\title{
DEVELOPMENT OF A TUNABLE 3D WRINKLED PLATFORM FOR DESIGNING CELLULAR THREE-DIMENSIONAL COMMUNICATION NETWORKS
}

\author{
by \\ Bethany R. Hughes, B.Eng
}
A thesis presented to Ryerson University
in partial fulfillment of the
requirements for the degree of
Master of Applied Science
in the Program of
Chemical Engineering

Toronto, Ontario, Canada, 2016

CBethany R. Hughes 2016 
Development of A Tunable 3d Wrinkled Platform for Designing Cellular ThreeDimensional Communication Networks MASc in Chemical Engineering 2016

Bethany R. Hughes Department of Chemical Engineering Ryerson University

\section{Author's Declaration}

I hereby declare that I am the sole author of this thesis. This is a true copy of the thesis, including any required final revisions, as accepted by my examiners.

I authorize Ryerson University to lend this thesis to other institutions or individuals for the purpose of scholarly research.

I further authorize Ryerson University to reproduce this thesis by photocopying or by other means, in total or in part, at the request of other institutions or individuals for the purpose of scholarly research.

I understand that my thesis may be made electronically available to the public. 


\begin{abstract}
The study of cell-cell communication is hindered by the absence of a platform which is capable of specifically directing cellular growth while allowing examination of the communication between cells. In this thesis, a tuneable micro-to-nano scale wrinkled nonplanar platform was developed and optimized through the use of photolithography and a microfluidic washing method. The platform demonstrated the ability to create micro and nanowrinkled structures in a wide range of flow conditions. The developed platform was then used as a cell culture platform to investigate the spacing dependence of bovine fibroblasts. The identification of a critical bridging distance for bovine fibroblasts provided a means to optimized the platform for culturing a cellular communication network between bovine fibroblasts. The cellular network which resulted demonstrated, via FRAP (Fluorescence-recovery-after-photobleaching), the capacity for communication between cells. Creating multilevel length scaled structures on a tunable platform which directed cellular growth while maintaining communication presents potential applications in research, industry and clinical aspects.
\end{abstract}




\section{Acknowledgements}

The presented work herein would not have been possible without the assistance of several people.

I would like to thank Dr. Dae Kun Hwang and Dr. Stephen Waldman for their time and expertise that went into all the work behind this thesis and also their outstanding supervision, guidance, support and encouragement in both my research and my development as a scientist and engineer. I would also like to acknowledge the assistance of Seyed-Shahriar Riazi for not just one but two summers of assisting with this project.

Furthermore, I would like to thank all my lab mates in both the Waldman and Hwang labs: Dehi Joung, Imran Sheikh, Dr. Johanna Weber, Ken Choi, Kristina Collavino, Dr. Lorraine Chiu, Dr. Minggan Li, Olena Bojchuk, Omar Subedar, Roberto Tarantino, Seyed Riazi, and Warren Tang for creating such a great atmosphere in the lab. A big thank you to all my friends in Toronto, P.E.I. and the rest of Canada. Especially Alex, Michelle, Imran, Kelly and my canine companion Fawkes who are always there for me.

My sincerest thanks go to my parents for always supporting and believing in me, for embedding the confidence in me that all things turn out well eventually. Thank you so much! 


\section{Table of Contents}

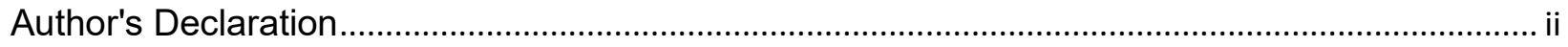

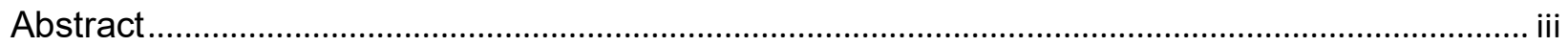

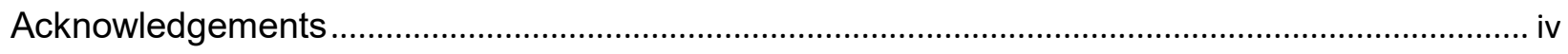

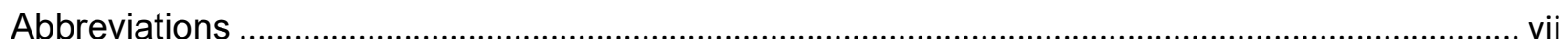

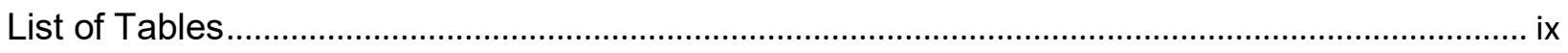

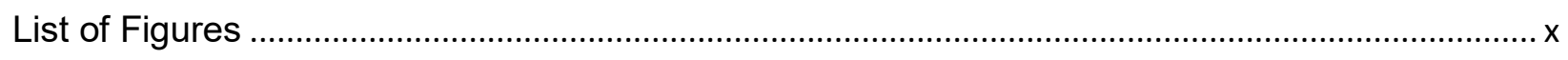

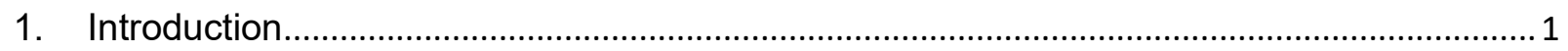

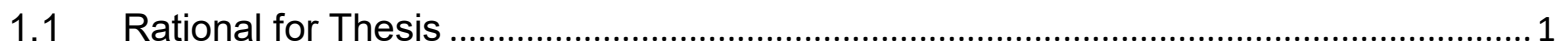

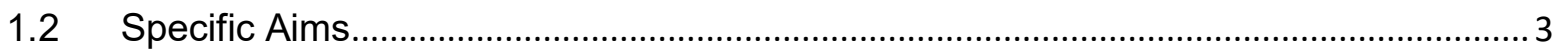

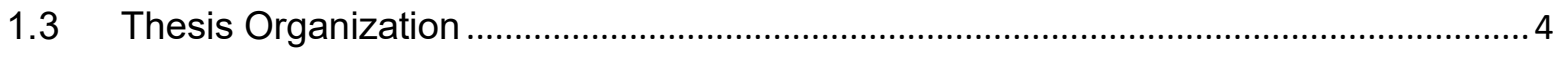

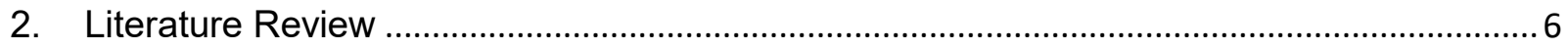

2.1 Topographical Features for Cell Culture ............................................................... 6

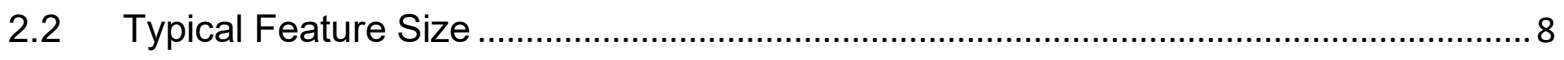

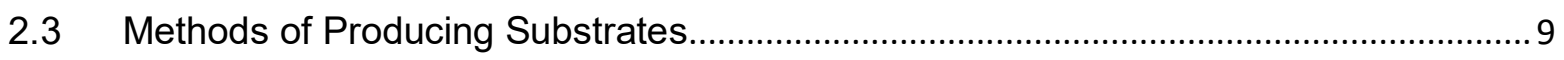

2.4 Current Work in Micro and Nano Topography to Influence Cellular Behavior............15

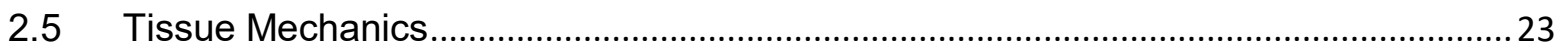

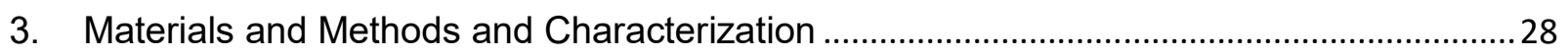

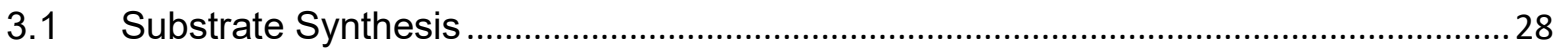

3.2 Substrate Cleaning for Cell Culture …................................................................... 33

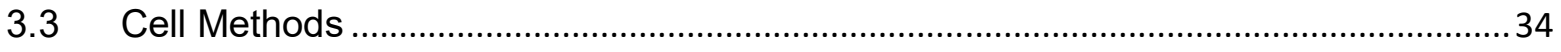




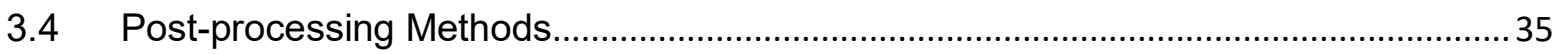

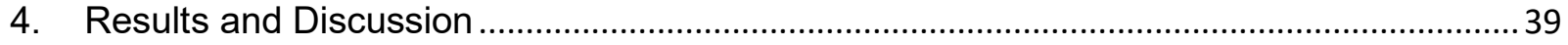

4.1 Nonplanar Cell Culture Environment to promote cellular network formation ..............39

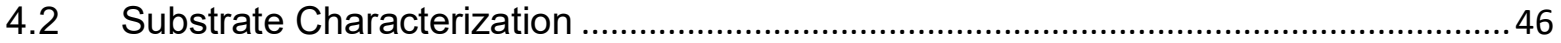

4.3 Cellular Response to Post Topography …................................................................ 49

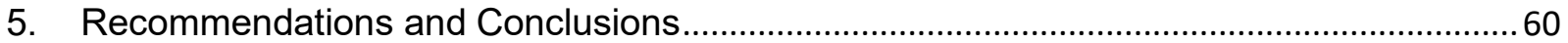

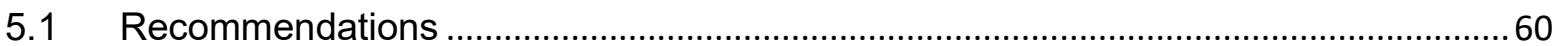

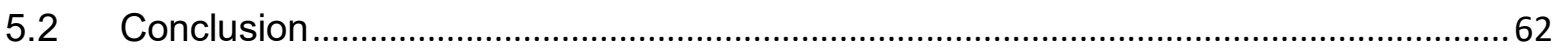

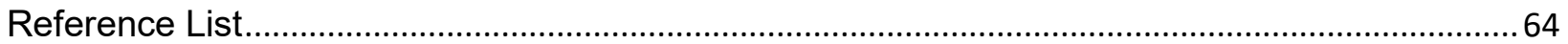




\section{Abbreviations}

\begin{tabular}{ll} 
Abbreviation & Full Name \\
$\mu \mathrm{m}$ & Micrometer \\
2D & Two Dimensional \\
3D & Three Dimensional \\
dH2O & Distilled water \\
DMEM & Dulbecco's Modified Eagle Medium \\
ECM & Extra Cellular Matrix \\
FRAP & Fluorescence recovery after photobleaching \\
HEPES & N-2-hydrocyethylpuperazine-N'-2-ethanesulfonic acid \\
hr & Hour \\
min & Minute \\
mL & Mililiter \\
mm & Milimeter \\
mM & Millimolar \\
NaOH & Sodium Hydroxide \\
nm & Nanometer \\
PBS & Phosphate Buffered Saline \\
PCPL & Partially Cured Polymer Layer \\
PDMS & Polydimethylsiloxane \\
PEG-DA & Poly(Ethylene)-Glycol Diacrylate \\
Sec & Second \\
\hline
\end{tabular}


Ultraviolet 


\section{List of Tables}

Table 2.1: Range of feature sizes for patterned substrates with cell culture …….......................... 9 


\section{List of Figures}

Figure 2.1: Traditional Cell Culture System Classification .........................................................

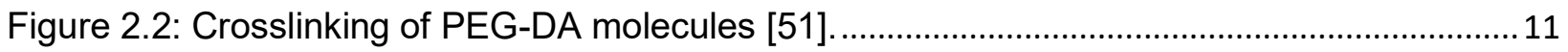

Figure 2.3: Cross-section of a microchannel demonstrating the limited photolithography[55]...13

Figure 2.4: Depth of field for a microscope influences the shape of objects polymerized under

UV light due to the profile exhibited [51].

Figure 2.5: Relationship between the monomer concentration and the UV exposure time used to

create the surface and its resulting wavelength [61].

Figure 2.6: Geometry classifications of non-planar surfaces. A) Post, B) Pit, C) Groove 16

Figure 2.7: (A) Boundary between posts and a planar surface and the effects of post size on fibroblasts interaction [17] (B) Smooth edges of flat culture vs ruffled edge of micropatterned culture [34] (C) Neuron attachment to a nanopost silicon substrate [3] (D) rounded microposts with additional of nanoparticles [62](E) microscaled wrinkles on microposts [54] (F)Wrinkled post array has a similar morphology to a leaf scale on a yellow pitcher plant [61] (G) [63] $(H)$ Measuring contractile forces within a single cell using microposts [35].

Figure 2.8: Fibroblast response to varied pit sizing (Diameter:Spacing, $\mu \mathrm{m}$ ) [31]. Large pit diameters allowed the fibroblasts to remain entirely inside the pit while smaller pit diameters were passed over by the fibroblasts 20

Figure 2.9 (A) Neurite extension with varied channel width [29] (B) Neurite extension in larger channel widths [28] (C) Pitted microgrooves, a hierarchy structure fabrication [76] (D) Fibroblasts on wrinkled surfaces demonstrate a alignment compared to flat surfaces [77] (E) nano-wrinkles to control cell behavior showing the nanowrinkle structure on the bottom and a flat vs wrinkled cell response above [18] (F) Curved microgroove structure [16] (G) Average cell length increases over time on a planar surface [78]......

Figure 2.10:Schematic of cell attachment mechanism [10] 
Figure 2.11: $(A)(B)$ native staining of gap junctions in fibroblasts of a ligament, the cells line up

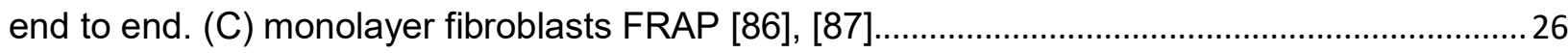

Figure 2.12: Schematic example[le of the FRAP process...........................................................27

Figure 3.1: A thin layer of PEG-DA is chemically bonded to a glass microscope slide. Using photolithography an array of microposts is created on the surface of the PEG-DA layer. ..........29

Figure 3.2: Chemically bonding of PEG-DA to a glass microscope slide [94]..........................30

Figure 3.3: Preslide Prep: Forming the PEG-DA foundation layer by sandwiching PEG-DA between glass cover slides 31

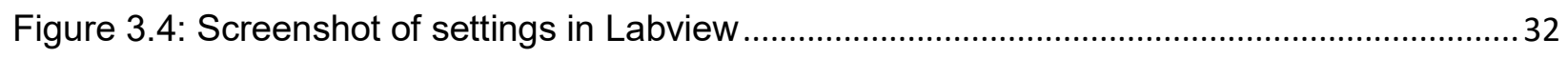

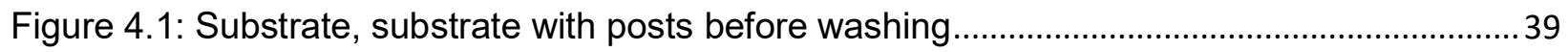

Figure 4.2: (A) Diffusion method setup; (B) Results from diffusion method.................................. 40

Figure 4.3: (A) Droplet method using $500 \mu \mathrm{L}$ of ethanol dropped via a pipette............................ 41

Figure 4.4: Characterization of Drop Method. All with 2 drops. ................................................ 42

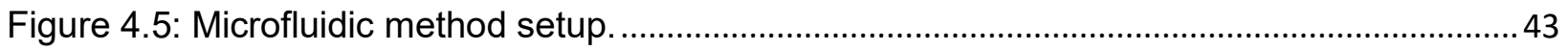

Figure 4.6: Visualization time capture of microfluidic post washing method using Rod-B with fluorescent video capture.

Figure 4.7: Characterized microfluidic method, posts have repeatable tunable wrinkle morphology. Scale bar $5 \mu \mathrm{m}$.

Figure 4.8: Comparison of nonselective vs selective nano-pattern. A) nanoscaled geometry appears uniformly across entire surface [62] B) wrinkles only appear on the nonplanar geometry .45

Figure 4.9: Summary of focusing effect in channel on post structure. .47

Figure 4.10: Characterization of microfluidic washing to achieve a tuneable platform via washing time or flowrate. 48 
Figure 4.11: Transition zones containing bio-regional wrinkles $(A)$ micro to nano transition $(B)$ nano to smooth transition 49

Figure 4.12: Cell experiments show cells ignore smooth posts but prefer to attach to wrinkled posts (A) SEM images show cells spread on substrate and ignore the smooth posts. (B) A representative SEM image shows that bovine fibroblasts attach to wrinkled posts after 3 days' cell culture and they climb onto the posts and conform to them, forming cell wrapped posts.....50 Figure 4.13: SEM images show varied post spacing distances and the effect of the spacing on

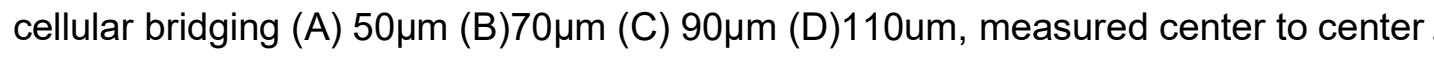
.51

Figure 4.14: Trends for post spacing and cellular attachment .52

Figure 4.15: Polarity of fibroblasts A)microscale wrinkled planar surface maintains unipolar fibroblasts [77] B) flat planar surface presents multipolar fibroblasts [77] C) bridging fibroblasts maintain unipolar morphology D) non-bridging fibroblasts demonstrate a multipolar morphology.

Figure 4.16: Immunostaining of bovine fibroblasts bridging posts vs on a planar surface. Staining for actin vinculin nucleun phallodin. The focal adhesion complexes can be observed at random points on the planar surface. However on the microposts they are present only on the post. .55

Figure 4.17: cells spread actin cytoskeleton stained formed stress fibres between dots, dots of microcontact printing of ECM protein, B16 cells - mouse melanoma cells. gold coated coverslip, inked with hydrophoblic alkanethiol . [101]. At a distance of $25 \mu \mathrm{m}$, spreading was limitedand cells became triangular, ellipsoid or round. Post spacing of 50um. .56

Figure 4.18: FRAP (Fluorescence recovery after photobleaching) demonstrates basic communication exists scale bar $=25 \mathrm{um}$. Red circles is areas where laser was applied for photobleaching 
Figure 4.19: FRAP Fluorescence Intensities for Region 1 being the photobleached cell and Region 2 being the non-photobleached cell.

Figure 5.1: Potential new photomask design to create posts with a distance between the rows to prevent bridging, greater than $90 \mu \mathrm{m}$, and in each row less than $90 \mu \mathrm{m}$ between each post. This would lead to lines of cells. 


\section{Introduction}

\section{$1.1 \quad$ Rational for Thesis}

The three dimensional (3D) microenvironment is the foundation for a cell and has been recognized to significantly influence cellular behavior in comparison to cells anchored to planar (2D) surfaces[1]. To mimic the in-vivo microenvironment, cells are typically placed in an artificially created extracellular matrix (ECM) with physiological conditions similar to their native tissues. This microenvironment can be broken down into physical and chemical features which both play a significant role in cellular growth. The majority of cell culture research performed has been divided into specific areas of study. Topics such as: cell-matrix interactions, mechanotransduction, intracellular signaling, biomechanics, and biochemistry need to be combined to take the next step forward in cell culture and exploit these mechanisms[2]-[4]. The study of these topics is hindered by the absence of a platform which is capable of specifically directing cellular growth while allowing examination of the communication between cells [5].

The topographical basis for directing cellular behavior is through the physical environment surrounding the cells. Many authors have demonstrated over the past decade that topographical features including microgrooves by Dalby et al., microposts by Dowell-Mesfin et al., and micropits by Petersen et al. influence the cellular response [6]-[8]. Physical cues can be achieved through molding, adding material, removing material, or a combination of all of the above [9]. Topographical cues can be on the microscale and nanoscale; and each scale can create a specific sought after response [5]. Thus, it is well documented that contact guidance produces changes to cellular fate processes [9]. The physical link between the specific anchorage points in cells with the 
extracellular environment are called focal adhesions [10]. Generally, focal adhesions pull on the ECM and substrate which the cell is attached to [10]. The cell can sense the resistance of the material which causes changes in cellular processes depending on the type of cell and the resistance it senses [10].

Nature has evolved many systems over hundreds of millions of years with sophisticated and complex designs which provide far superior characteristics than current artificially possible, even with today's technologies [11]. Orientation and alignment of cells and their surroundings at each level in a hierarchical structure give tissues their specific properties and are responsible for the exceptional characteristics which have been observed in biological systems. Wrinkled formations are used extensively as a tool for superior surface characteristics and increased efficiency for processes such as energy intake. Numerous scales of wrinkled morphology exist, however, most examples of wrinkled morphology exist on non-planar surfaces. Plant leaves, sea corals, seeds, pollen grains and epithelial tissues all exhibit some form of wrinkled morphology. Despite the prevalence of wrinkles in nature, integration of wrinkled morphology into biological platforms remains rudimentary. Two dimensional wrinkled surfaces can be produce using a number of techniques, but creating nonplanar 3D features with wrinkled morphology has remained challenging. Some bottomup bulk techniques could potentially produce such a platform. However, these approaches are time consuming with limited material choices.

Following orientation and alignment of cells, the next step is to understand the inter-cellular communication required for continued cell response. Cellular communication occurs throughout all cells of a living system. However, particular 
subsystems rely extensively on direct communication to function such as neuron synapses and ligaments. Whole-section samples and more-detailed, structural/mechanistic studies are the techniques used for the majority of studies on cellular communication [12]. An advantageous improvement in research methods for cellular communication would include the ability to direct cellular morphology while exhibiting controlled cell-cell communication [5].

\subsection{Specific Aims}

A cells microenvironment has a direct effect on the regulation of cellular behavior. In tissue engineering, modifying the microenvironment of a substrate has the global objective to induce a desired cellular function. Literature has demonstrated the effects of micro- and nano- patterned biomaterial platforms to control cellular alignment and organization. Thus, with substrate modification a biomaterial can be tailored to conduct, induce, or block tissue responses. However, many current surface topographies studied examine the effect for directing cellular growth but with a resultant cellular behavior observed on these structures similar to that on planar, two-dimensional surfaces. Three dimensional interactions between cells and/or their extracellular matrix are required to truly mimic cellular function and behavior in vivo.

This thesis aims to help be an initial step towards creating organized cellular networks. In addition to providing a fabrication method of a tunable dynamic cell culture platform. To achieve these objectives, studies were conducted as follows:

- Development of a novel tunable nonplanar cell culture system

- Explore fibroblast bridging in response to the developed culture system

- Investigate the potential for cellular communication network designing 


\subsection{Thesis Organization}

Within five chapters this thesis provides an introduction to the area of research, identification of gaps in current literature and a summarization of the experiments and results obtained.

The Literature Review discusses the complex hierarchical organization of natural tissues and the factors which control the cellular microenvironment. In addition, current fabrication techniques used for modifying the microenvironment for tissue engineering are summarized briefly. Lastly, this section describes various applications of micro- and nano- topography in tissue engineering.

Materials and Methods provides all necessary and relevant information for reproduction of all data in this thesis. This covers synthesis of the entire cell culture platform, all cell culture techniques used and the process steps performed for each of the post-process imaging and analysis methods.

Results and Discussion begins with a summary of the novel tunable cell culture platform including the development and characterization which are then sequentially used to create all substrates for cellular experiments. Then results are presented from all cell culture experiments performed including topography effect, critical bridging distance and cellular communication testing of bovine fibroblasts. The importance of the observed results including the advantages and disadvantages of different methods for the novel platform fabrication, the significances of the bridging effect, the incorporation of cellular communication and potential applications are discussed throughout this section. 
Finishing with the Conclusion which will summarize the entire thesis, identify any limitations of the work performed and suggest additional and future work to be performed. 


\section{Literature Review}

\subsection{Topographical Features for Cell Culture}

Classically, geometry has been used to define cell culture systems. A large body of research exists relating to structure fabrication techniques which produce improved substrates for cellular culture. Systems can be classified as either surface or bulk structures based on geometry. A surface is defined as having material in contact with only a portion of a cell. This can be referred to as heterogeneous for extracellular material. However, a bulk system is defined as having an extracellular material surrounding the cell on all sides or homogeneous. Both of these classifications can be further broken down by their geometric structure, as outlined in Figure 2.1. Surface structures can be divided into planar and non-planar geometries. Planar surfaces cover the extensively used flat culture system currently used in scientific research while nonplanar geometries exist as surfaces with material modification through addition or subtraction methods. However, a non-planar surface still provides material on only a portion of the cell membrane. Bulk structures are more complex and generally can be classified as linear structures, porous structures, beads or suspension. These bulk structures are the traditional geometries considered when discussing three-dimensional culture systems. Further classification is based upon dimensional effects such as size range, porosity, and stiffness. However, when length scales and new fabrication methods are combined together, classification becomes difficult due to heterogeneity of the structures. 


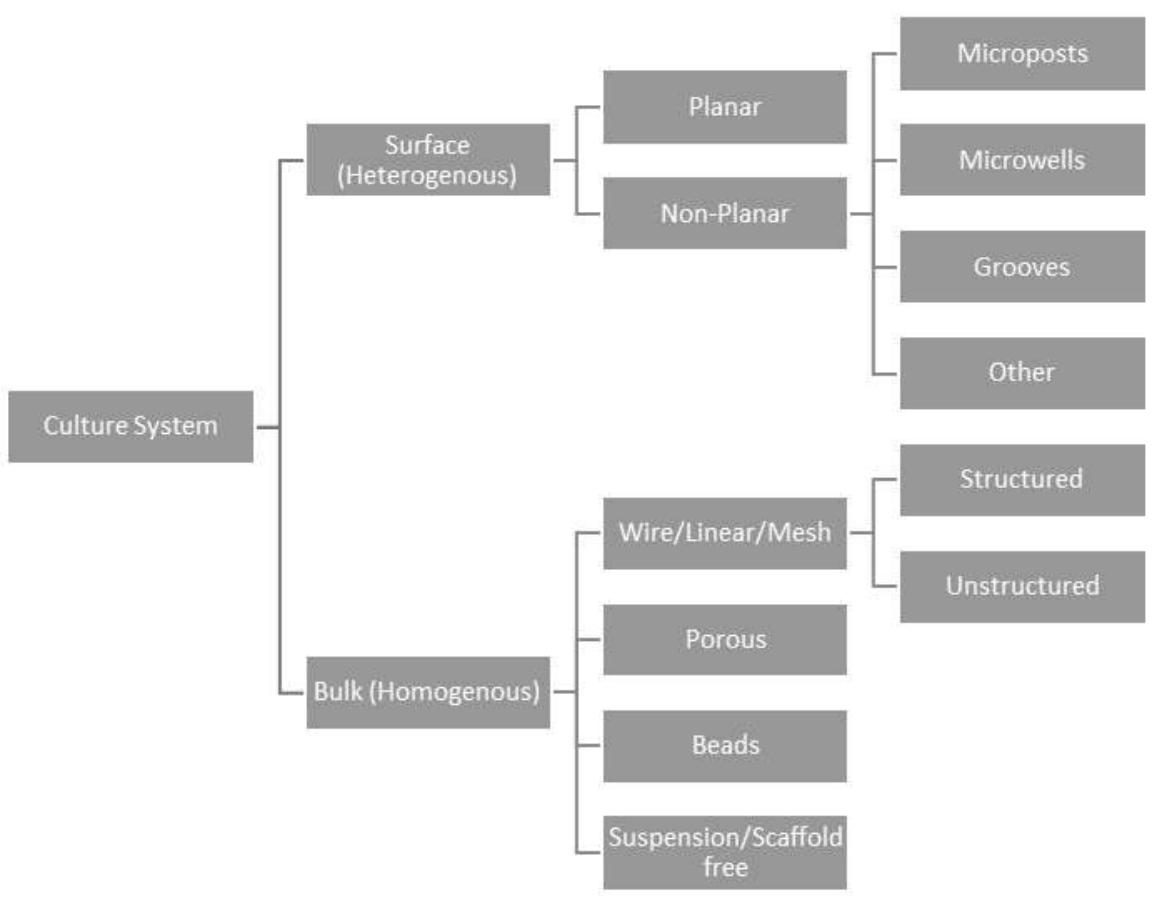

Figure 2.1: Traditional Cell Culture System Classification

An important aspect across all culture systems is the implementation of different length scales, lotus-type superhydrophobic surfaces for self-cleaning, anti-icing and dew collection all exhibit hierarchal structures responsible for their properties [13]. Length scales have an enormous effect on what relative forces are at play. Different length scales that cannot affect each other are decoupled and make it possible to examine specific problems without considering the whole. Each scale contains different mechanisms which all play a role in how the material properties are expressed and how the body reacts to the material [9]. To deliver a functional tissue one must examine the material structure at a supra-cellular $(>100 \mu \mathrm{m})$, cellular $(10-100 \mu \mathrm{m})$, and subcellular $(<10 \mu \mathrm{m})$ length scale [9]. At the subcellular level biological recognition becomes important and cell-surface receptor-ligand binding effects must be examined [9]. At the 
cellular length scale many material properties exhibit effects, such as: pore size, micropattern, roughness, cell-cell and cell-matrix interactions [9]. The supra-cellular level is responsible for host integration with the particular material being introduced, and the resulting mechanical properties, biocompatibility, and cellular ingrowth is determined at this level inside the structure [9]. This hierarchical structure is responsible for all structures. For example, orientation and alignment of cells and their surroundings at each level in a hierarchical structure give tissues their specific properties and is responsible for the exceptional characteristics which have been observed in biological systems. It is important to consider all levels on this scale down to the molecular interactions to be able to predict and direct cellular fate [14].

A problem which has been largely overlooked by many studies and techniques when considering platforms for cell culture is the aspect of multiple length scales in a native 3D environment. Majority of the fabrication methods utilize planar surfaces and are not extendable into a 3D environment. Very few studies have achieved fabrication processes which allow the planar fabrication method to continue to be developed in a non-planar environment [15], [16]. Furthermore, even fewer studies have proceeded with cellular culture. The future of these surfaces is non-planar structure and the majority of these techniques are not extendable into a 3D environment.

\subsection{Typical Feature Size}

The range of sub-cellular structures used in recent literature is summarized in Table 2.1. Throughout these papers, it is evident that every cell type requires a different microenvironment in terms of feature type, size and shape. For instance, fibroblasts did not interact with microposts shorter than $2 \mu \mathrm{m}$, whereas, neuron outgrowths interact 
with microposts of $1 \mu \mathrm{m}$ [3], [17]. This demonstrates that the surface must be designed specifically for the desired cell type, which is currently not employed in cell culture. Additionally, Loseberg et al. reported control of fibroblasts as depths as shallow as 35 $\mathrm{nm}$ [18]. Thus, differences observed within the same cell type indicated additional factors at play such as surface chemistry and surrounding biomolecules. Additionally, with advancing fabrication techniques the size range continuously decreases.

Nanoscaled environments, such as wrinkles are governing cellular effects [18], [19].

Thus the surfaces of these patterns used in cell culture need to examine the effects of their nanoscaled surface properties.

Table 2.1: Range of feature sizes for patterned substrates with cell culture

\begin{tabular}{|c|c|}
\hline Feature & Size Range \\
\hline Grooves & 1-100 $\mu \mathrm{m}$ wide $\times 1-20 \mu \mathrm{m}$ deep [6], [20]-[29] \\
\hline Wells & $<100 \mu \mathrm{m}$ diameter [8], [30]-[33] \\
\hline Posts & $\begin{array}{l}\text { Height: } 90 \mathrm{~nm}-25 \mathrm{um} \text {, Typically around } 2-10 \mu \mathrm{m} \\
\text { Diameter: } 200 \mathrm{~nm}-100 \mathrm{um} \text {, Typically } 5-10 \mu \mathrm{m} \\
\text { Spacing: } 4-70 \mu \mathrm{m} \text { [7], [17], [34]-[39] }\end{array}$ \\
\hline
\end{tabular}

\subsection{Methods of Producing Substrates}

The first use of topographical cues in cell culture was reported in 1914 by R. Harrison [40]. Harrison et al. cultured embryonic frog cells onto spider web demonstrating that the cells elongated at the fibers [40]. However, it was not until 1998 when George Whitesides adapted soft lithography techniques to create substrates for biological applications that research into the underlying mechanisms of such 
phenomena's were established [41]. Although other physical properties can effect cellular behavior such as tension, this thesis will focus on topographical properties [42]. The first and most common techniques used to generate well defined structures at the microscale and nanoscale is photolithography [43].

\subsubsection{Photolithography Background}

Lithography is a process used in microfabrication to create patterns with features down to a few nanometers on parts of a thin film or the bulk of a substrate. Masked lithography uses masks to create patterns over the entire surface of a substrate. Photolithography utilizes UV light exposure to transfer a geometric pattern from a photomask to a light-sensitive polymer on the substrate. The UV light is initially passed through a mask which patterns the radiation onto the polymer which provides the selectivity exposure. A cross-link is a bond that links one polymer chain to another (covalent or ionic) which causes a loss of the ability to move as individual chains, creating a solid/gel [44]. When a bulk polymer is crossed-linked, it means the whole section of the polymer has been exposed to a crosslinking technique. The degree of crosslinking can be adjusted with multiple variables. Lower crosslink density reduces the viscosity of solutions or creates a gel/gum polymer. Increasing the crosslink density proportionally increases the stiffness of the resulting material and can create more rigid solid structures [45]. Many sources of energy can initiate the chemical reactions that form crosslinked bonds such as heat, change in $\mathrm{pH}$, pressure, or radiation (UV light for example). A hydrogel is a gel in which with the addition of water the gel swells. Hydrogels are hydrophlilic and highly water absorbent, and maintain a high water content, thus they possess a degree of flexibility very similar to natural tissues. The high 
water content and tunable properties resemble native extracellular matrix (ECM). Common hydrogel polymers are PolyHEMA (2-hydroxy-ethyl methacrylate), ethylene glycol dimethacrylate (EDMA), MMA (methylmethacrylate), and isobutyl methacrylate, Poly(ethylene)-glycol-diacrylate (PEG-DA). PEG-DA has previously been used in tissue engineering, cell culture, drug delivery systems, and biosensors demonstrating is biocompatibility [46]-[49] . PEG-DA is a crosslinking hydrogel that when exposed to UV light in the presence of a photoinitiator can crosslink to form a structured solid gel with mechanical properties similar to tissues [50].

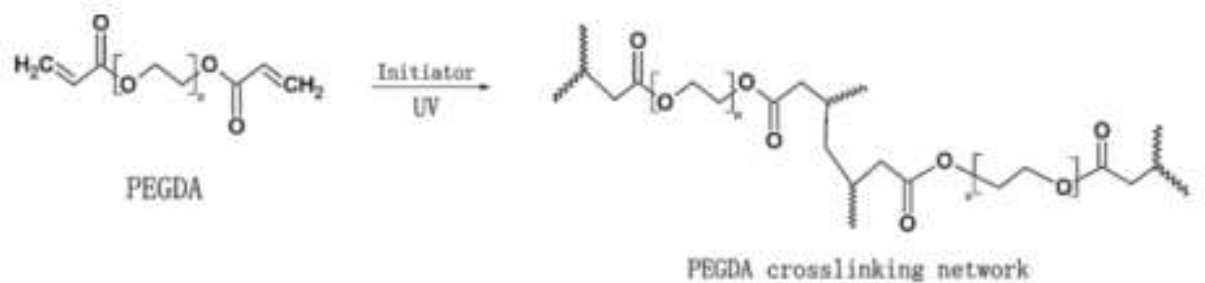

Figure 2.2: Crosslinking of PEG-DA molecules [51].

The crosslinking of monomer chains occurs through a process called free-radical polymerization. Free-radical polymerization is a type of chain-growth polymerization where polymers form by the addition of free radical building blocks repeatable. The free radical building blocks involve initiator molecules. Monomer units add to the initiating free radical and result in the growing of polymer chains. This mechanism of polymerization is divided into the following steps: initiation, propagation, and termination. Initiation begins with an initiator molecule which contains bonds that easily break forming an initiator fragment with an unpaired electron [52]. The initiator is chosen depending on the type of energy to be used to break the initial bond. For example, a photoinitiator is used for UV light activation such as Benzoin, Benzil, or Benzophenone. Molecules with unpaired electrons are called free radicals. This free radical attacks the 
double bond of the monomer to form a bond and thus propagate the free radical and a lengthen molecule which can react with another molecule to create an even longer polymer. An active polymer chain spends most of its time increasing the length of the chain, or in this propagation mode. This propagation does not continue indefinitely. Eventually, two free radicals collide together or other impurities and end the chain. Oxygen is a common inhibitor and produces an oxygen radical which is significantly less reactive. This immediately stops the growth of the two chains and produces the final molecule. The final polymer structure will be a mixture of molecules of different sizes. As the mask provides the geometric shape to be replicated, when UV light comes in contact with PEG-DA it causes polymerization. If a PDMS (Polydimethylsiloxane) microchannel is added to the polymerization path, a phenomenon occurs at the interface between the top of the channel, PDMS, and the polymerizing PEG-DA. The PEG-DA is prevented from polymerizing to the walls. The walls are comprised of PDMS which is gas permeable. In a system open to the air, oxygen is present at the interface of PDMS and PEG-DA. When PEG-DA becomes a radical, the oxygen from the surrounding air, reacts before other PEG-DA chains, becoming a stable, uncrossedlinked layer [53]. Thus, the height of the PDMS channel provides the limiting factor for the height of the formed PEG-DA structure. However, if the bottom of the PDMS microchannel is nonpermeable, oxygen cannot diffuse through, creating structures on the surface. This results in one side of the extruded structure to remain attached to the surface, Figure 2.3 [54]. 


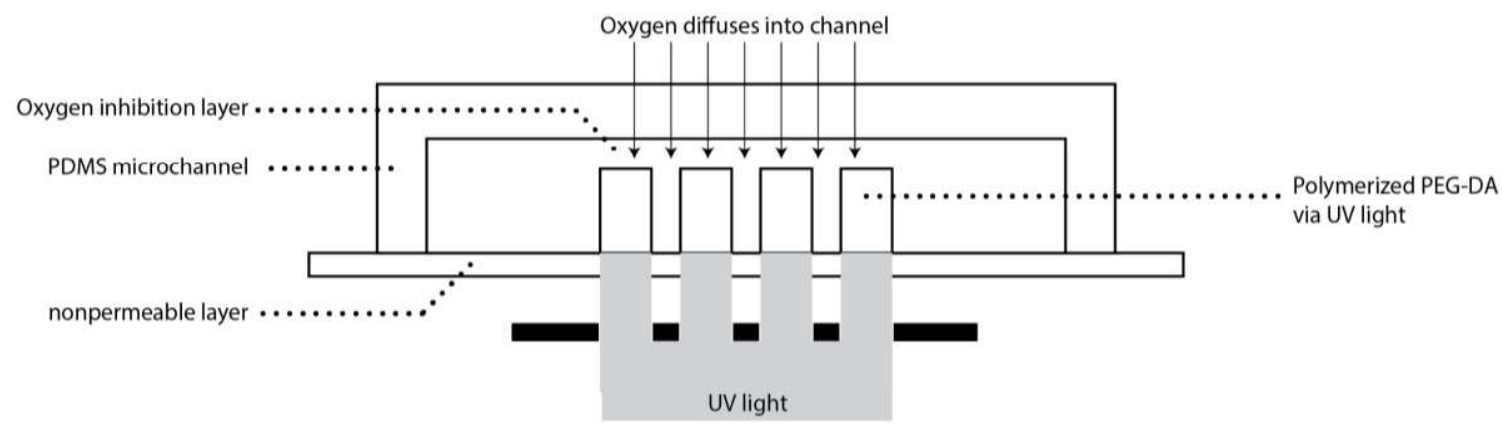

Figure 2.3: Cross-section of a microchannel demonstrating the limited photolithography[55].

A significant factor for determination of height and shape from a channel is depth of field during polymerization. Depth of field is the range of object distances where the object maintains a satisfactory sharp focus, Figure 2.4 . The application of depth of field to the microscope objective provides the basis for the shape of the posts. Using the in focused section of the light, the pattern provides straight, nonconical structures [56]. In addition, the UV light experiences radial decline in intensity, which causes the shape of the top of the posts [56].

a
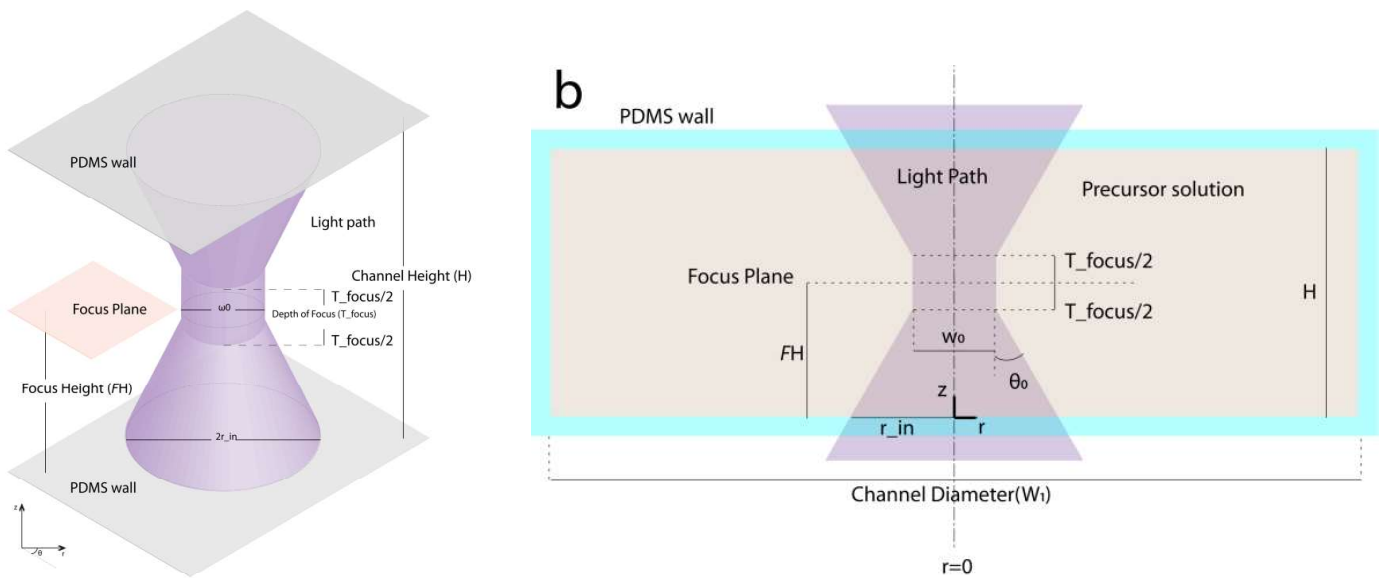

Figure 2.4: Depth of field for a microscope influences the shape of objects polymerized under UV light due to the profile exhibited [51]. 


\subsubsection{Creating Wrinkles with Plasma}

Excellent reviews exist which discuss the detailed mechanics of this surface wrinkling [57]-[60]. A brief explanation, begins during the photopolymerization step. A partially cured layer of the polymer is created on the cured body [54]. Oxygen is present in the prepolymer solution and inhibits polymerization until the oxygen removed by UV induced free radicals [53]. This is a gradual depletion and causes a continuous diffusion of oxygen from the PDMS channel. This results in polymerization of the polymer to begin from the glass slide where oxygen cannot diffuse. The polymerized polymer structure continues to grow with a partially cured outer layer. When the UV source is removed, polymerization stops. The resulting structure is defined by the photomask used by a cured body and still contains a partially cured layer.

This layer contains a network of partly crosslinked PEG-DA mixed with uncured monomers. The thickness of this layer is regulated by UV dose. Some of the uncured monomer can be removed post-polymerization, however, some will still remain trapped in this layer.

When this layer is exposed to plasma, a wrinkled microstructure results. Charged ion that make up plasma cause additional crosslinking to occur in this partially cure layer creating a thin crust. However, this also results in expansion of the polymer. As the plasma ions penetrates deeper into the partially cured layer it introduces in-plane compressive stresses in the crust, forming wrinkles [53].

The stiffness of the PCPL (Partially Cured Polymer Layer) can be modified by UV exposure time, which determines the concentration of remaining monomer concentration and as a result modifies the stiffness of the material. A long UV exposure 
causes more crosslinking between monomers and results in less unconverted monomers remaining in the layer. By increasing the exposure time from $300 \mathrm{~ms}$ to 700 ms, Li et al. demonstrated a decrease in the unconverted monomer from $40 \%$ to $6 \%$ respectively [54]. At 2 seconds, no monomers remained unconverted. Following exposure to plasma, the amount of unconverted monomers was proportional to the resulting wavelengths of wrinkles produced, Figure 2.5 [54].

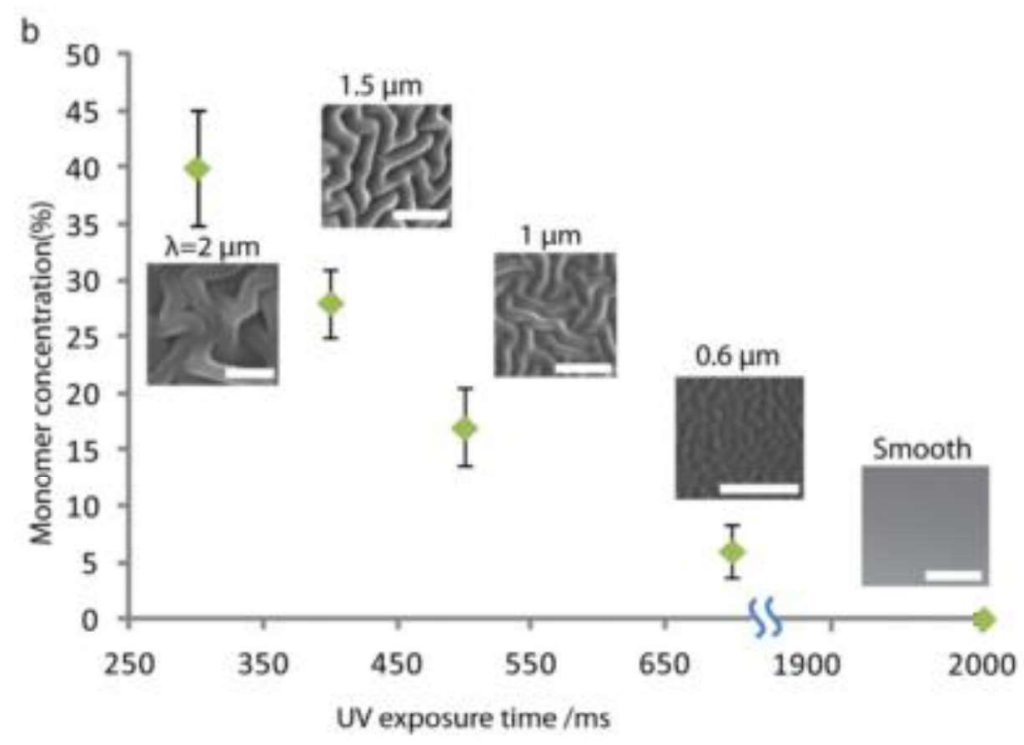

Figure 2.5: Relationship between the monomer concentration and the UV exposure time used to create the surface and its resulting wavelength [54].

\subsection{Current Work in Micro and Nano Topography to Influence Cellular Behavior}

\subsubsection{Surface Culture Systems}

Research into surface topography can be sub-classified into three leading structures: posts, pits and grooves (Figure 2.6). A post is an upright protrusion from the substrate. A pit is a depression into or completely through the substrate. Lastly, a groove is a channel, as such, these structures are significantly longer in one direction 
than in the other two directions. The majority of studies have used the groove microstructure because these structures possess the simplest manufacturing process.

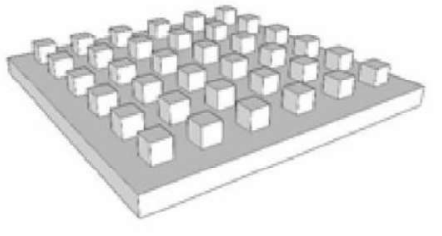

A

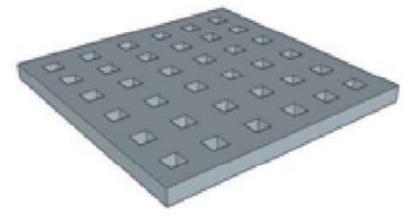

B

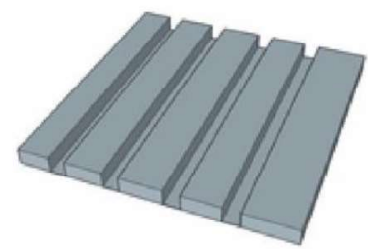

C

Figure 2.6: Geometry classifications of non-planar surfaces. A) Post, B) Pit, C) Groove Posts Related Systems

Ghibaudo et al. fabricated microposts from molded PDMS to investigate adhesion and migration of fibroblasts [17]. By varying the inter-post spacing and the diameter of the posts, changes in cellular morphology and actin stress fibers were observed. They also reported increased cell motility on the patterned substrates, in addition to fewer actin stress fibers [17]. Cellular morphology was longer and less flat on the patterned areas of the substrates when compared to non-patterned, Figure 2.7a. Additionally, cellular morphology differed depending on the perceived in interpost spacing by the fibroblast cells. For posts that were close together, the cell body remained on the top of the pillars with thin projections of up to $50 \mu \mathrm{m}$ protruding from the cell body, Figure 2.7a [17]. For shorter posts fibroblasts attached both on top of the pillars and on the surrounding surface, engulfing whole posts, Figure 2.7a [17]. It was noticeable that cells on posts with relatively low aspect (height to diameter) ratios exhibited morphology similar to flat surfaces [17]. 


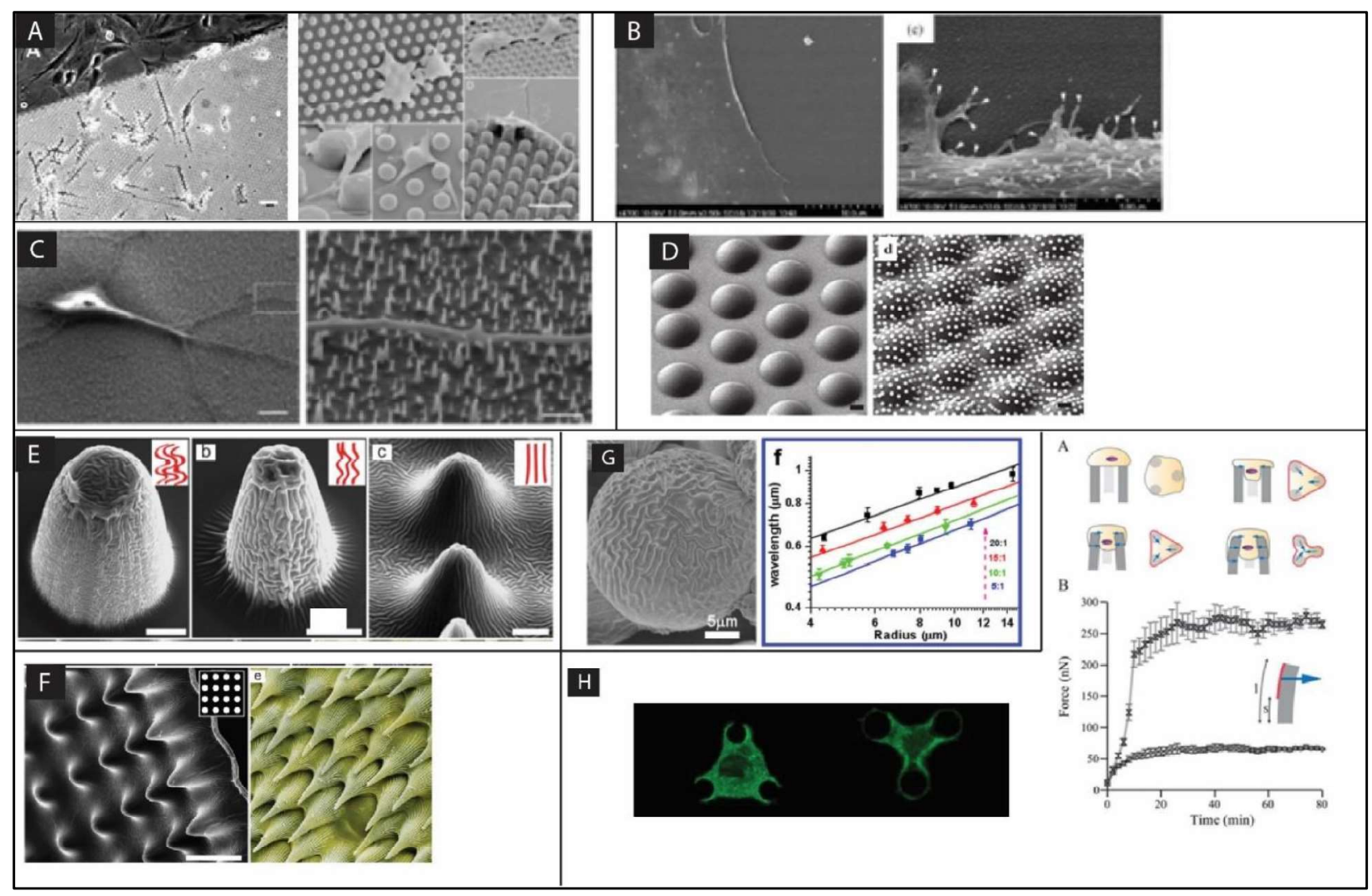

Figure 2.7: (A) Boundary between posts and a planar surface and the effects of post size on fibroblasts interaction [17] (B) Smooth edges of flat culture vs ruffled edge of micropatterned culture [34] (C) Neuron attachment to a nanopost silicon substrate [3] (D) rounded microposts with additional of nanoparticles [61](E) microscaled wrinkles on microposts [54] (F)Wrinkled post array has a similar morphology to a leaf scale on a yellow pitcher plant [54] (G) [62] (H) Measuring contractile forces within a single cell using microposts [35]

In contrast, Berry et al. demonstrated fibroblast interaction with nanoposts 40 $90 \mathrm{~nm}$, with approximately an aspect ratio of 2:1 [34]. They found the substrates caused the cellular morphology to be ruffled with filopodia interactions around all edges of the cell body,Figure 2.7b [34], [63]. Further examination of the filopodia extensions showed that these features terminate on top of a post, Figure 2.7a. Berry et al. also concluded that no significate difference was shown when comparing 40 vs $90 \mathrm{~nm}$ post-cell interactions [34]. 
Ghislain et al. explored the effect of randomly placed nanoposts on neuron elongation and axon differentiation [3]. The nanoposts were fabricated with reactive ion etching of silicon substrates. This resulted in posts $700 \mathrm{~nm}$ in height and a radius of 35 $\pm 17 \mathrm{~nm}$. The outgrowths from the cell body remained on the top of the posts with approximately $500 \mathrm{~nm}$ between adhesion sites Figure 2.7c [3]. In comparisons to neurons grown on flat surfaces, Ghislain et al. reports fewer neurites, faster elongation and earlier differentiation of an axon of cells grown on this nanopost substrate [3].

Li et al. created tunable wrinkled morphology on a micropost,Figure 2.7E [54]. These structures were similar to leaf scales of a yellow pitcher plant which uses the structures to trap insects, Figure 2.7F [54]. Another, microwrinkled nonplanar structure was created on microbeads Figure 2.7G [62]. Patterning method for nonplanar surfaces, Bhawalkar et al. fabricated rounded microposts with addition of nanoparticles, Figure 2.7D [61]. A common use of microposts is to measure the contractile forces within a cell Figure 2.7H [35].

Wrinkles are commonly known to enhance both appearance and performance of surfaces. Wrinkles in nature are used extensively to increase energy intake or surface area and create superior surface characteristics such as high flexural stiffness [64]. From plant leaves, sea coral, and pollen grains to epithelial tissues and paint finishes [54], [64], wrinkles play a significant part in life processes, often on a length scale that is so fine the unaided eye cannot detect them.

A number of techniques exist to create artificial wrinkles based around mechanical instabilities of a compressed thin film. A common approach is to create a pre-strained foundation then deposit a thin layer of stiff materials [54], [65]-[68]. 
Modification of top layer to create a disparity from the bottom layer then occurs using many sources of external energy such as fire, ion etching, plasma, electrocehmical polymerization, UV ozone [69]-[74]. Then the material is released of the pre-strained foundation and results in a compression of the top layer. This results in a wrinkled surface. Another strategy to create surface wrinkles is modification of the surface layer without a pre-strained foundation. The mechanisms involved in this strategy cause the top surface layer to swell due to a solvent, ion beam, plasma, laser, or interfacial energies [54]. Both mechanisms are able to produce tunable patterned wrinkles. Numerous scales of wrinkled morphology exist; however, most examples of wrinkled morphology exist on non-planar surfaces. Despite the prevalence of wrinkles in nature, integration of wrinkled morphology into platforms remains reasonably undeveloped.

A technique previously developed in Dr. D Hwang's lab to create spatially tunable and controllable wrinkles on 2D/3D microstructures surfaces aims to increase usability of wrinkled morphologies in emerging applications. However, this technique has been previously limited to the micro length scale.

\section{Pit Related Systems}

Pitted geometries have also demonstrated some level of cellular control, Figure 2.8. Berry et al. created an array of pits ranging from $7-25 \mu \mathrm{m}$ diameters with spacing's of either 20 or $40 \mu \mathrm{m}$ [31]. The largest diameter pits allowed the fibroblasts to enter the pit, regardless of spacing [31]. They hypothesized that the radius of curvature was a factor which the cell could distinguish as a cell would not be able to determine the diameter of a pit prior to being inside the pit [31]. Time lapse microscopy showed that 
the cells are able to exit the pits once they entered [31]. Pits smaller than $7 \mu \mathrm{m}$ allowed cells to pass over the top [31].

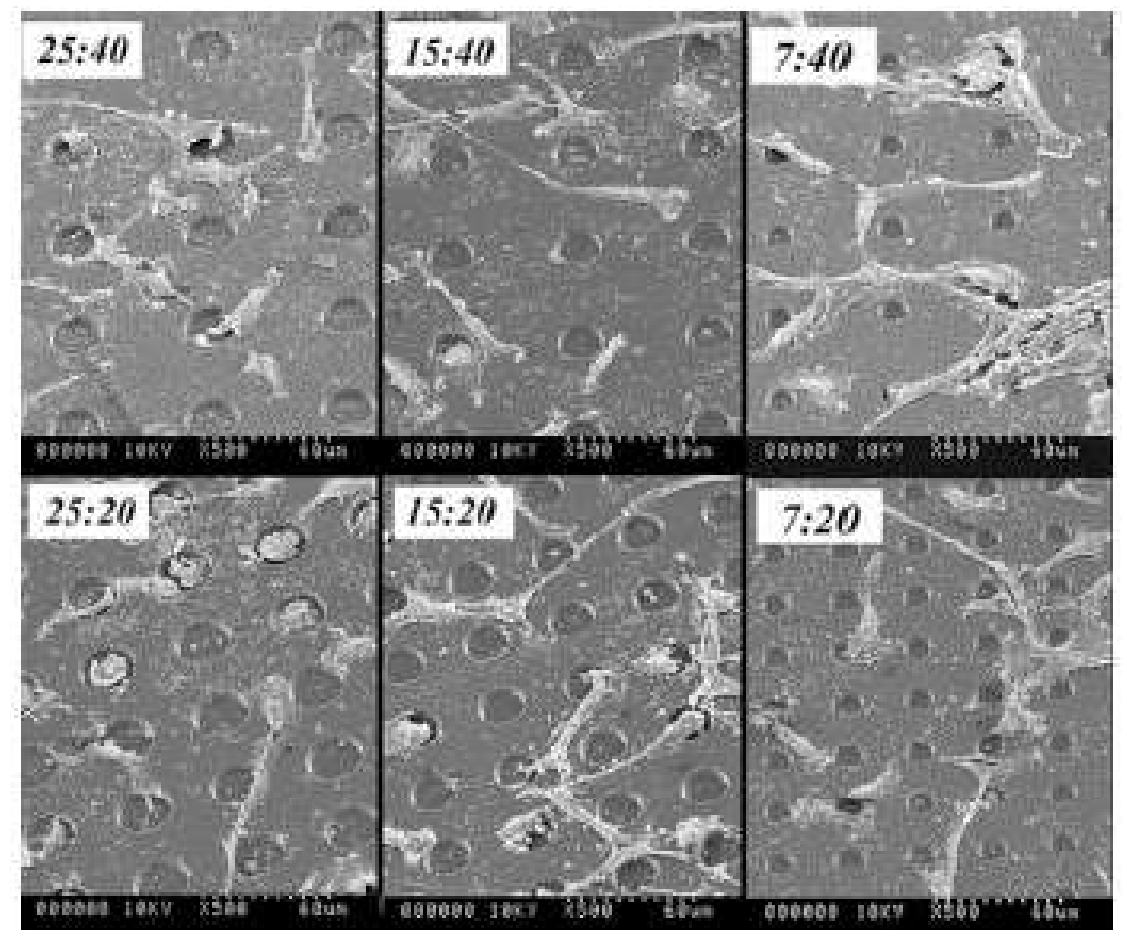

Figure 2.8: Fibroblast response to varied pit sizing (Diameter:Spacing, $\mu m$ ) [31]. Large pit diameters allowed the fibroblasts to remain entirely inside the pit while smaller pit diameters were passed over by the fibroblasts.

\section{Groove Related Systems}

Beduer et al. used microgrooves to study the long term effects of patterned substrate growth on neurons [29]. Molded PDMS substrates of multiple channel widths (Figure 2.9A) were used to identify a characteristic width which resulted in an ideal growth [29]. Beduer et al. concluded that their micropatterened substrate are effective for neuron guidance [29]. Comparison between immunocytochemical staining demonstrated a high differentiation rate with evident neurite alignment along edges of the micro-channels (Figure 2.9A). Channel widths larger than the soma had differentiation rates comparable to flat surfaces [29]. However, channel widths smaller 
than the soma diameter caused reduced differentiation and development of the stem cells into neurons [29]. As a result, the number of neurites per neuron was affected by channel width and increased with wider channels [29]. They suggest that neurites require open space to elongate [29]. Thus, cells confined in the small channels only grow neurons along the channel [29].

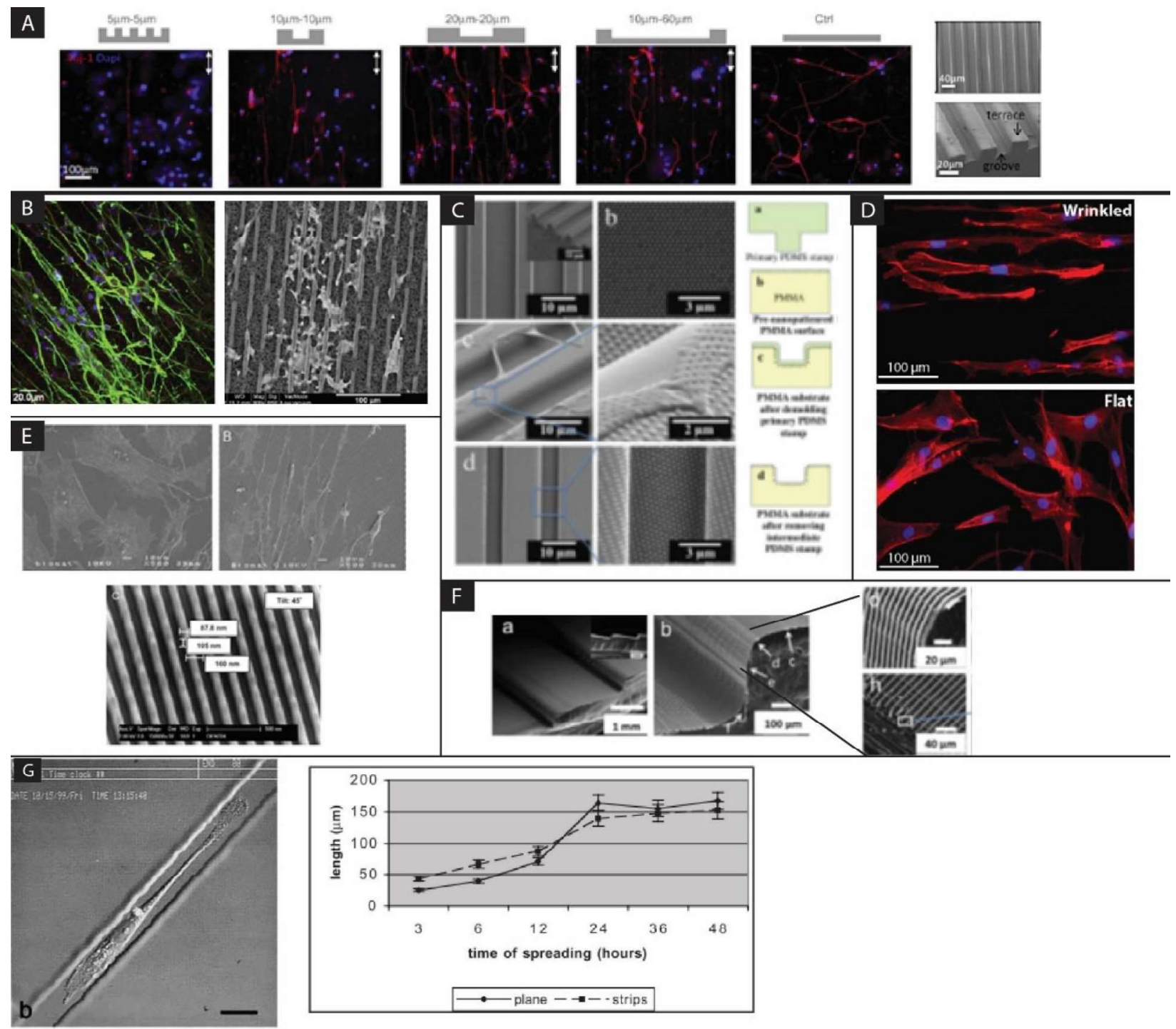

Figure 2.9 (A) Neurite extension with varied channel width [29] (B) Neurite extension in larger channel widths [28] (C) Pitted microgrooves, a hierarchy structure fabrication [75] (D) Fibroblasts on wrinkled surfaces demonstrate a alignment compared to flat surfaces [76] (E) nano-wrinkles to control cell behavior showing the nanowrinkle structure on the bottom and a flat vs wrinkled cell response above [18] (F) Curved microgroove structure [16] (G) Average cell length increases over time on a planar surface [77] 
Similar results were achieved by Morelli et al. where microchannel were used to improve the guidance of neurite extension resulting in improved orientation and alignment [27]. Overall, they explored larger channel widths (Figure 2.9B) which produced less individual cellular guidance than Beduer et al. reported [27]. Overall, elongated neurites oriented parallel to the grooves. Differences in morphological behaviour between fibroblasts cultured on smooth and nanogrooved substrate was also observed (groove depth: 5-350 nm, width: 20-1000 nm), Figure 2.9E [18].

One study was able to fabricate nanopitted microgrooves by a two step approach [75]. First a naonpatterned surface was created, followed by a second step of stamping a microgroove pattern in the PDMS, Figure 2.9C [75]. A second fabrication method obtaining nonplanar platform was implemented in a similar two step approach. Microchannels were created on a planar surface, followed by manipulation of the surface into a curved shape Figure 2.9F.

Another example of cellular alignment leading to the formation of basic tissue structure was observed in cardiomyocytes. When Cardiomyocytes are aligned they produce aligned sarcomere structures and can generate action potentials [78]. Wrinkled planar surfaces have been shown to aligned fibroblasts (Figure 2.9D) however, communication has not been examined on these surfaces [76].

Levina et al. examined the maximum length of fibroblasts. The fibroblasts reach a maximum length after a 24 hour period and was independent of the micropatterned presented, Figure 2.9G [77]. However, other cell types such as epithelial cells became longer with cultured on grooved surfaces and appeared to not be regulated by their length [77]. 


\subsection{Tissue Mechanics}

Cellular adhesion is the molecular basis for assembling cells into solid threedimensional tissues. Tissues are composed of cells and extracellular matrix (ECM). The majority of cells are inherently adhesive and attach to their surroundings including neighboring cells and surrounding ECM [79]. This anchorage dependence is required for survival in many cell types [79]. Literature has begun to establish the role of cell-cell and cell-ECM interactions in the cellular fate processes, which includes differentiation, proliferation, migration, and apoptosis [80], [81]. In addition to cellular fate processes, adhesion plays an important role is other biological processes such as morphogenesis, tissue regeneration, wound healing and gene regulation [10], [81]. Abnormalities in cellular attachment have been linked to disease such as carcinogenesis, thrombogenesis, and abnormal embryogenesis [81].

The physical link between the specific anchorage points in cells with the extracellular environment are called focal adhesions [10]. Generally, focal adhesion spots pull on the ECM and substrate which the cell is attached to [10]. The cell can sense the resistance of the material which causes changes cellular processes depending on the type of cell and the resistance it senses, illustrated in Figure 2.10 [10].

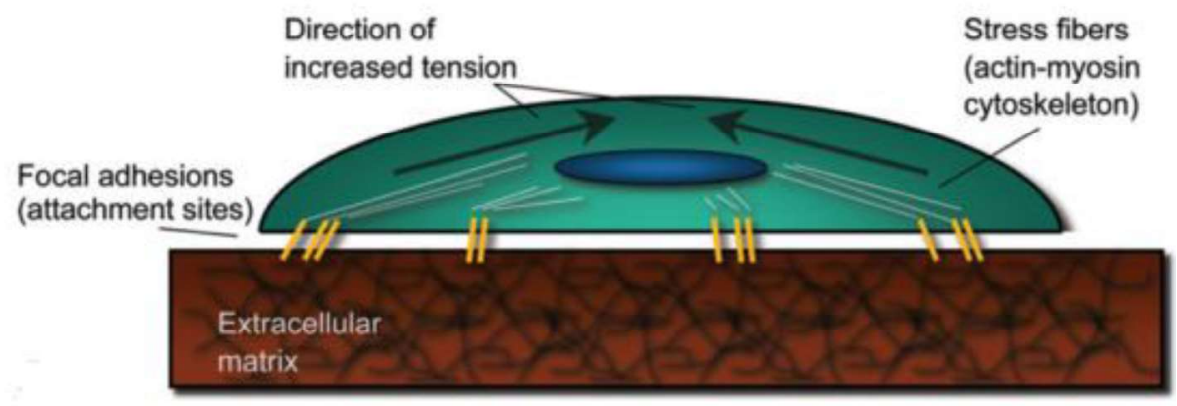

Figure 2.10:Schematic of cell attachment mechanism [10] 
Focal adhesions are connected to the ECM via specific cell adhesion molecules in the cellular membrane. Cell adhesion molecules (CAMs) are specific molecules which act as a biological glue and are involved with transmitting the biomechanical and biochemical signals [82]. The location, number, and type of CAMs in a cells plasma membrane determine how a cell responds to its microenvironment [82]. CAMs are classified into four categories: immunoglobulin, integrin, cadherin and selectins [82]. Integrins are primarily involved with ECM binding, selectins bind to sugar moieties, and cadherins are responsible for cell-cell binding [82]. Cells can bind to the ECM through these transmembrane integrins [5]. Three types of integrin receptors have been identified: hemidesmosomes, focal contacts, and fibrillars contacts [80]. Integrins are the primary sensor for external mechanical properties in the cell surroundings [10]. Demidesmosomes are mainly found in epithelial tissues and the basement membrane to intermediate filaments inside the cell through integrins [83]. Focal contacts consist of paxillin and vinculin. Focal contacts connect actin in the cyctoskeleton to the ECM [83] . Fibrillar contacts contain tensin and similarly to focal contacts, create a mechanical linkage to actin located inside the cellular membrane [80].

Integrins bind to only a small portion of the larger ECM proteins [80]. This information allows scientists to mimic larger ECM proteins, such as fibronectin, with small peptide fragments such as Arginine-Glycine-Aspartic Acid (RGD) [80]. Allowing functionalization of surfaces to simulate the ECM with simple addition of small peptide fragments [80]. However, sometimes to elicit a signal, more than one peptide must be bound to the cell. For example, it has been hypothesized that a synergy site on fibronetctin requires two peptides, RGD and PHSRN, to elicit a signal through integrin 
$\alpha 5 \beta 1$ [84]. An integral feedback relationship exists between the number and the location of integrins and the force exerted on the cell through with the modification to the ECM by the cell [10]. Thus, both mechanical environment and soluble factors play a role in dynamic cell interaction processes [10].

It has been demonstrated that many integral feedback relationship exists between a cell and its environment. However, an very small amount of information has been published in regard to the under-lying mechanisms of cell sensing, intercellular signaling, or long-term in response to micro and nano patterned surfaces. Research has shown that by altering the dispersal of extracellular matrix (ECM) proteins or altering surface topography, it is possible to modification cell morphologies and behavior [5]. The amount of control of the geometric arrangement on cellular behavior largely varies [43].

\subsubsection{Cell-Cell Communication}

Cellular communication occurs throughout all cells of a living system. However, particular subsystems rely extensively on direct communication to function. Two types of intercellular communication that are directly related to this are juxtacrine and paracrine signalling. Paracrine signalling, is a local signalling route that requires the secretion of molecules that travel very short distances to reach their target cell. which plays very important roles in synaptic transmission and hormone secretion [9].

Juxtacrine signalling requires direct contact to occur. Direct contact communication relies on junctions, called gap junctions, which connect the cytoplasm of adjacent cells allowing cytoplasm to flow from one cell to the connecting cell. These junctions allow for many process to occur such as cellular recognition, embryonic 
development, and immune response [9]. For example, cardiac muscle cells form gap junctions to allow unison contractions to occur and epithelial cells when one cell becomes injured, other cells are notified. Ligaments are composed of fibroblasts which align end to end surrounded by collagen fibers, Figure 2.11A. Ligaments have been characterized to demonstrate they posse gap junctions Figure 2.11B.

\section{A}

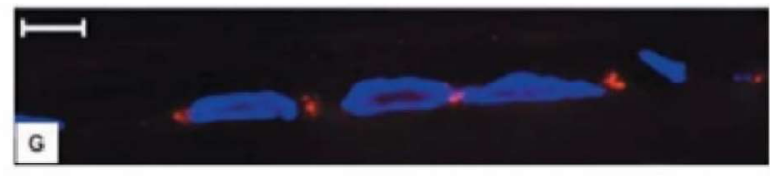

B
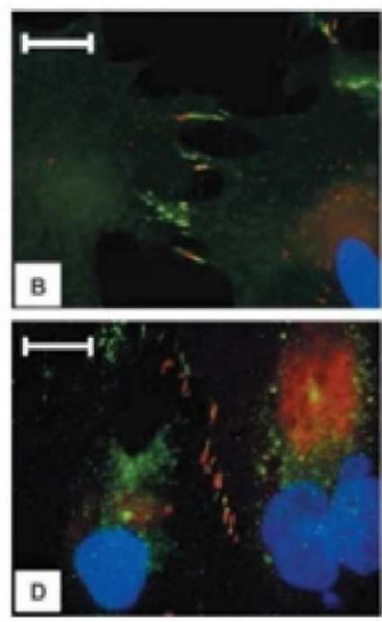
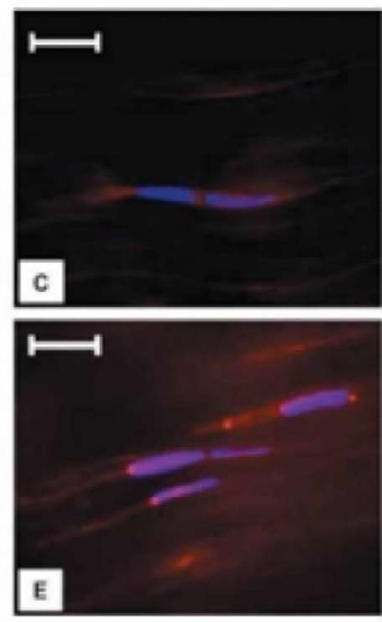

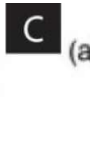

(b)

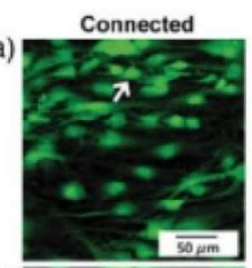

(b)

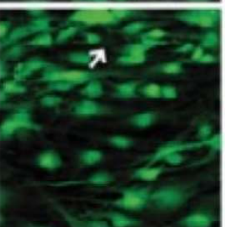

(c)

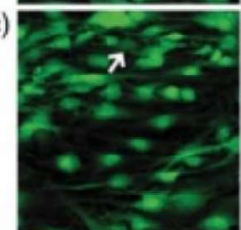

(d)

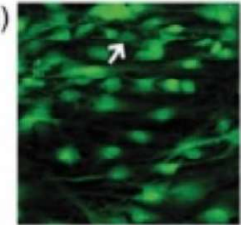

(e)

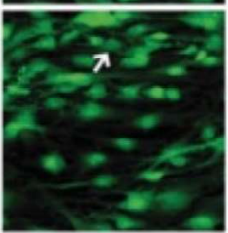

Isolated
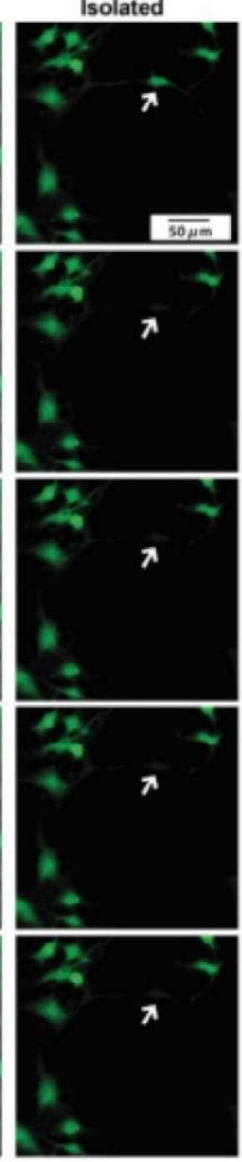

Figure 2.11: $(A)(B)$ native staining of gap junctions in fibroblasts of a ligament, the cells line up end to end. (C) monolayer fibroblasts FRAP [85], [86]

\section{Methods of Assessing Cell-Cell Communication via Gap Junctions}

Current methods of developing and analyzing a communication network between cells is very limited. Two methods of analysis exist, whole section samples and single cell assays. For example, for neuron analysis using single cell assays requires either 
specialized equipment or unusually large synapses models such as giant squid synapses, neuromuscular junctions, brain stems [87], [88]. Whole section samples from animals models are usually stained and imaged, in combination with molecule biology techniques to study specific molecules [87]-[89]. In addition to whole section sample analysis, communication can also be analysed using three types of assays metabolic coupling assays, electrical coupling assays and dye coupling assays [90]. Examples of these assays are microinjection, scrape loading, gap-fluorescence recovery after photobleaching (gap-FRAP), the preloading assay, and local activation of a molecular fluorescent probe (LAMP), or through measuring electrical conductance and metabolic cooperation [91].

Fluorescence Recovery after photobleaching (FRAP) is a technique that utilizes diffusion through a cell or part of a cell to quantify kinetics of a desired molecule. In the context of this study, FRAP was used to determine if communication exists between bridging cells. The basic principle behind FRAP is the application of a high powered laser onto a specific area causing the fluorescence molecules to become extinct and not refluoresce, called photobleaching, Figure 2.12. After a set amount of time the area that was photobleached is re-examined. If the area begins to fluoresce then the area is susceptible to diffusion from other fluorescence molecules.

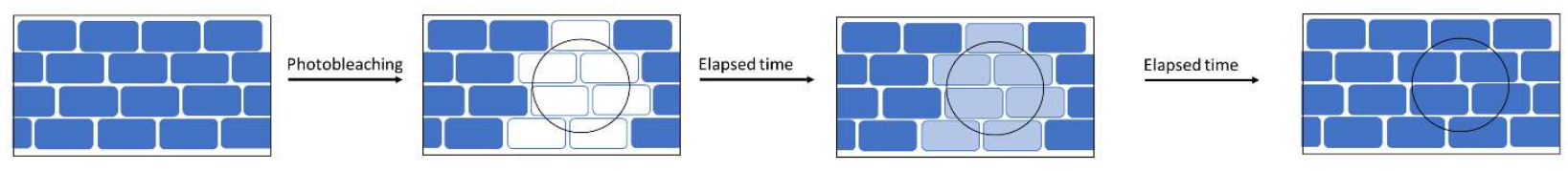

Figure 2.12: Schematic example[le of the FRAP process. 


\section{Materials and Methods and Characterization}

\subsection{Substrate Synthesis}

A topographical cell culture substrate was synthesised using photolithography of poly(ethylene)-glycol diacrylate (PEG-DA), a biocompatible hydrogel. Following fabrication, the substrate was cleaned and bovine fibroblasts were added to examine the effects of the culture system on cellular behavior. To analyse the effects, three imaging platforms were used. This chapter discusses in detail the protocol used to synthesis the hydrogel platform, the cell culture procedures and the imaging processing steps.

\subsubsection{Overall Substrate Structure}

The PEG-DA substrate was chemically attached to a standard microscope slide to prevent flipping and floating during cell culture and post-processing. The substrate consisted of a basement membrane of a PEG-DA layer with micropost protrusions fabricated of PEG-DA and polymerized to the basement membrane. 


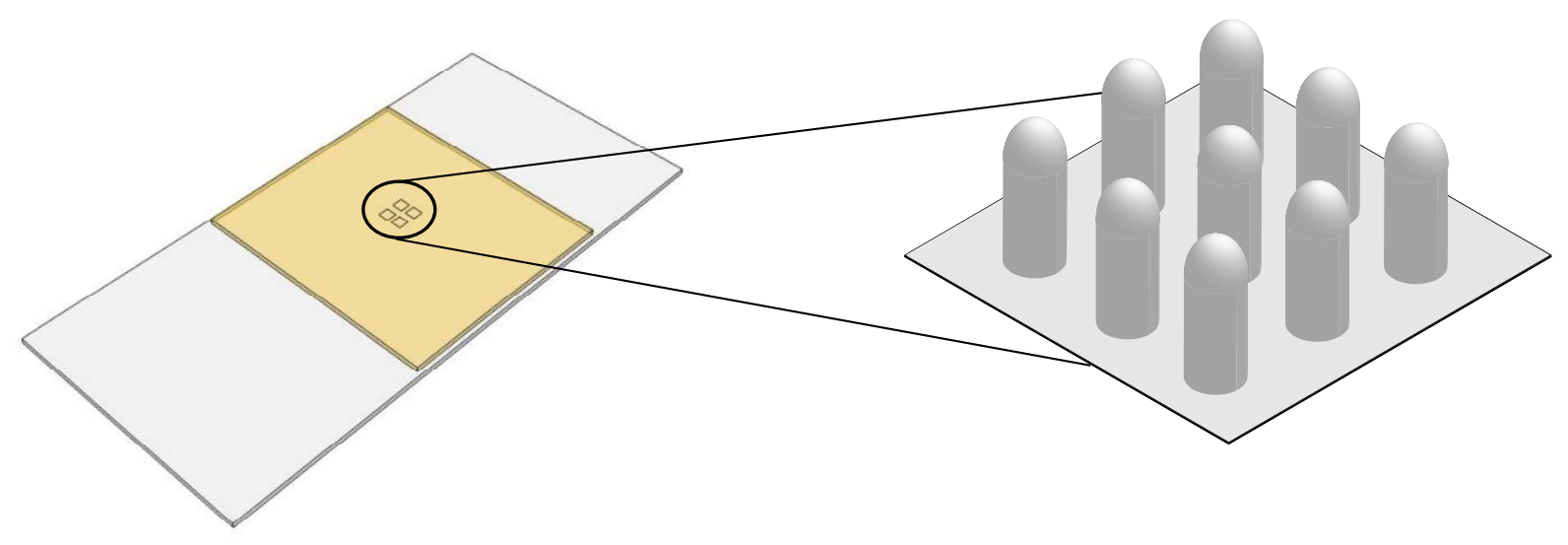

Figure 3.1: A thin layer of PEG-DA is chemically bonded to a glass microscope slide.

Using photolithography an array of microposts is created on the surface of the PEG-DA layer.

\subsubsection{Synthesis of Basement Substrate}

To prevent substrates from lifting during experiments, the glass microscope slide was treated with 3-(trimethoxysilyl) propyl methacrylate (MAPTMS) (Sigma Aldrich),Figure 3.2. 3-(trimethoxysilyl) propyl methacrylate is coupled with the surface by a reaction between the silane molecule and the oxygen on the surface [92]. This leaves the functional methacrylate unit free to crosslink with additional PEG-DA molecules. 


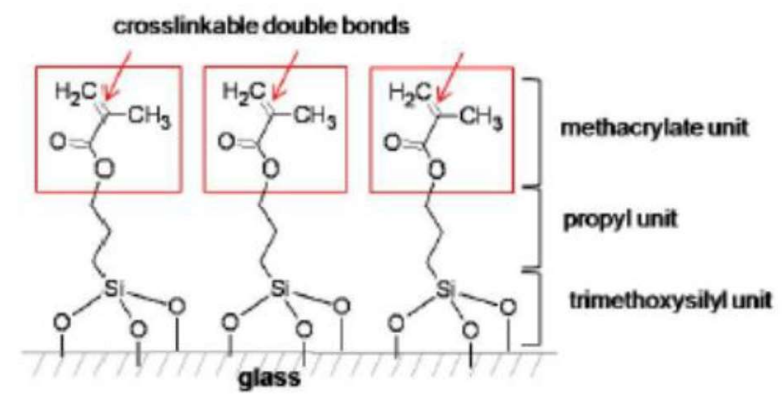

Figure 3.2: Chemically bonding of PEG-DA to a glass microscope slide [93].

The silanized cover slips were fabricated in the fume hood first by cleaning the glass cover slips with deionized water, followed by ethanol. The glass cover slips were placed in a container and let dry for 15 minutes to allow the ethanol to evaporate. In a glass container $200 \mathrm{ml}$ of $95 \%$ ethanol was measured. Then, $1 \mathrm{ml}$ of MAPTMS was pipetted into the ethanol and parafilm was placed over the top and mixed with a magnetic stirrer for 3 minutes. $6 \mathrm{ml}$ of the diluted 1:10 acetic acid was added to the ethanol-MAPTMS solution. The solution was continually stirred with the magnetic stirrer for an additional three minutes. The solution was poured on the glass cover slips in the containers and allowed to react with the glass cover slips for 3 minutes. The silanization solution was removed from the container and the glass cover slips were rinsed with ethanol 2 times to minimize the amount of residual reagents. The slides were allowed to air dry in the fume hood. The silanized surface is vulnerable to moisture and was immediately used to create a basement membrane of PEG-DA [93].

To create a layer of PEG-DA, a glass cover slip was set horizontally and $80 \mu \mathrm{l}$ of PEG-DA was pipetted onto the center of the cover slip. On each end, place another glass cover slide perpendicular to the bottom slip. Place a fourth cover slip on the top 
creating a sandwich, Figure 3.3. Allow expose for 2 days in the fluorescence light in the room.

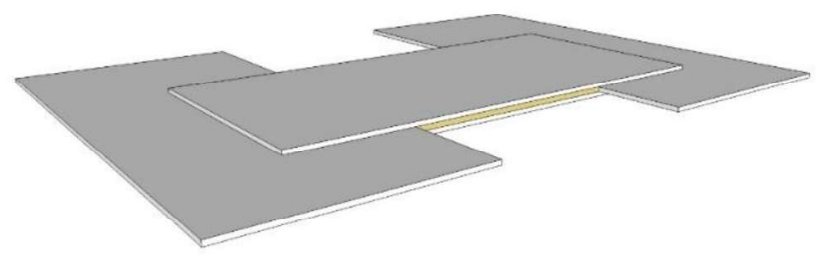

Figure 3.3: Preslide Prep: Forming the PEG-DA foundation layer by sandwiching PEGDA between glass cover slides

\subsubsection{Synthesis of posts}

\subsubsection{Photolithography}

Standard lithography techniques were used to fabricate a master silicon wafer. To make the PDMS channels from the master silicon wafer a silicone elastomer kit mix (PDMS, Sylgard 184, Dow Corning, Midland, MI, USA) was used. Using a 10:1 ratio, $55 \mathrm{~g}$ silicone and $5 \mathrm{~g}$ curer was placed into a disposable cup and mixed with a disposable pipette until the color goes opaque from bubbles (couple of minutes). Place the cup into vacuum dome, and allow air to be removed from the mixture $\sim 30-40$ mins until the liquid is clear again (bubbles all gone). Pour into the desired mold, cover and place in oven over night to gel. Cut out from PDMS mold by taking a scalpel and cutting in a circle around the circular silicon wafer. Remove PDMS from mold and place on a dark surface to cut out the individual channels. Cut channel inlets and outlets with a biopsy punch.

A Labview controlling application, previously created by Dr. Hwang's Laboratory, was used to control the shutter operation for the UV light control for an exposure time of $300 \mathrm{~ms}$. All of the settings are outlined in Figure 3.4. 


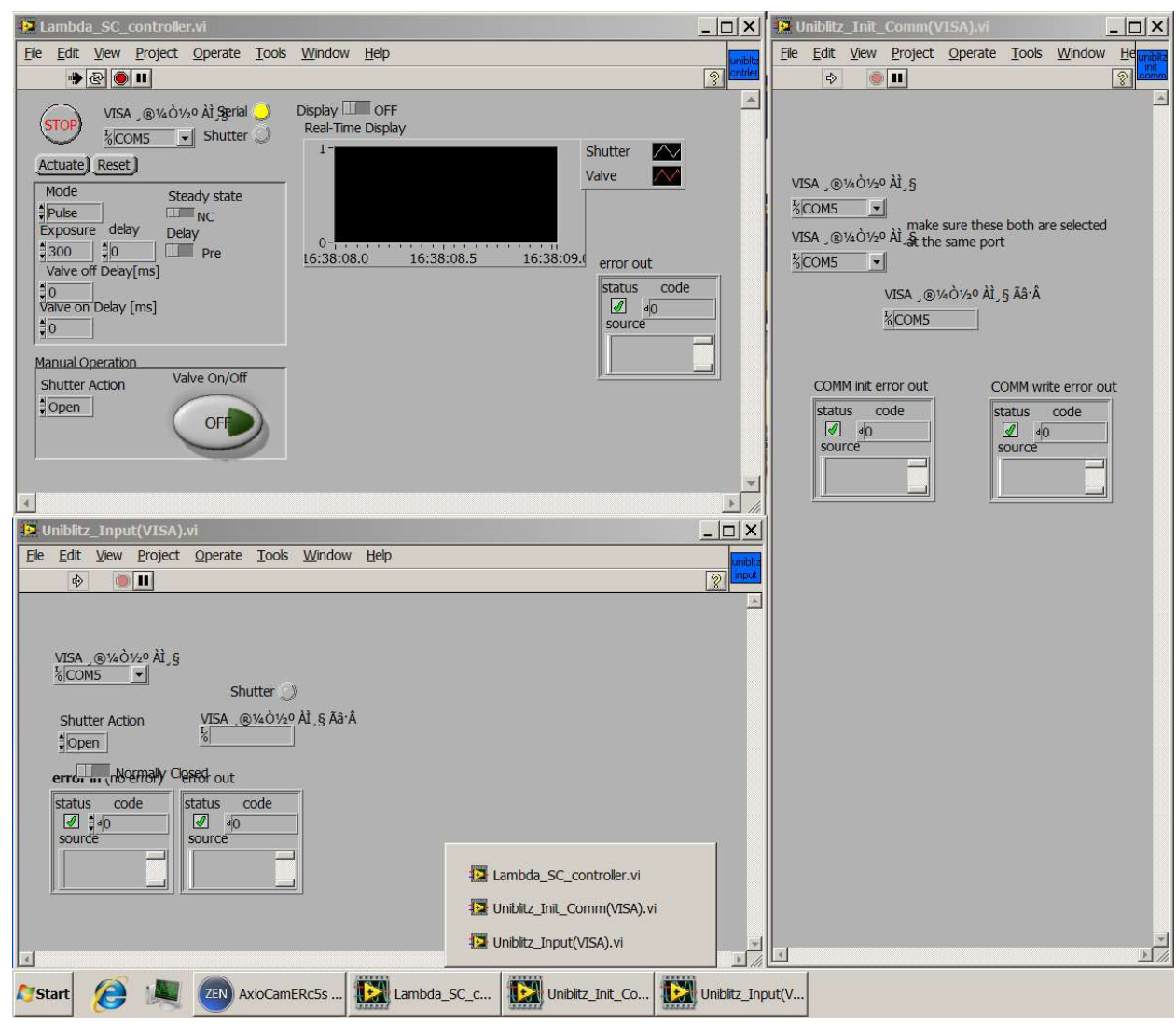

Figure 3.4: Screenshot of settings in Labview

Create PEG-DA solution by placing the following into a $1 \mathrm{ml}$ epindorph tube 950 ml PEG-DA 700 (95\%) (PEG-DA 700, Sigma-Aldrich), 50 ml photoinitator: 2-Hydroxy-2methyl propiophenone (5\%) (PEG-DA 700, Sigma-Aldrich). Mix the solution to by Vortexing. Fill PDMS channel with PEG-DA solution, place slide on microscope. Make sure microscope dial is on UV spectrum (not red, green or blue).

Locate the edge of channel. Use the large focus knob to bring the channel relatively into view. Use the fine focus so the edge of the channel creates the thinnest possible line. Then using the fine focus knob change the focus by $10 \mu \mathrm{m}$, see experimental results section, hit the 'actuate' button in Labview for polymerization to occur for $300 \mathrm{~ms}$. An ultraviolet (UV) light (305-390 nm) with intensity of $280 \mathrm{mw} \mathrm{cm}^{-2}$ 
(UV intensity meter, ACCU-Cal 50, CT) was used as the light source. The PDMS channel height was $40 \mu \mathrm{m}$ and used with the 10x objective. The depth of field for the setup was only $49.6 \mu \mathrm{m}$, solved in Equation 1, where the NA is the numerical aperture, Mag is the total magnification and the wavelength $(\lambda)$ is of the UV light [54]. This resulted in only $10 \mu \mathrm{m}$ margin to create complete posts. The importance of this was observed when determining the focus through the microscope in the photolithography process.

$$
\begin{gathered}
\text { Depth of field, } \mathrm{T}_{\text {Field }}(\mu \mathrm{m})=\frac{1000}{7 * N A_{\text {objective }} * M a g_{\text {total }}}+\frac{\lambda(\mu \mathrm{m})}{2 * N A_{\text {objective }}^{2}} \\
=\frac{1000}{7 * 0.3 * 10}+\frac{0.365}{2 * 0.3^{2}}=\frac{1000}{21}+\frac{73}{36}=49.65 \mu \mathrm{m}
\end{gathered}
$$

The next step is to wash the sample to remove any excess PEG-DA monomer. Multiple methods were utilized to determine optimum washing process, see experimental section below. Following removal of excess PEG-DA monomer, the samples were exposed to plasma (Harrick Plasma, PDC-32G, NY) for 30 seconds. This was done by placing the sample in the chamber, turn on the pump making sure the air valve is closed, allow pump to build up vacuum for $1 \mathrm{~min}$, turn on power (allow it to warm up for a few seconds), turn to high RF power, turn the three-way valve to the controlled air inlet and turn the knob until the color is dark pink inside. After 30 seconds, slowly release the vacuum by turning the three way valve all the way to the right. Examine the final structure under light microscope and store in a large petri dish.

\subsection{Substrate Cleaning for Cell Culture}

Substrates were placed in 6 well cell culture plates and soaked in $100 \%$ anhydrous ethanol for 2 hours (as an introduction of the hydrogel to a fluid environment 
which facilitated a slower swelling speed). Then replaced with $70 \%$ ethanol, repeated for 3 changes in the first 2 hours and then left overnight to sterilize the surface and remove any unpolyermized polymer.

\subsection{Cell Methods}

\subsubsection{Bovine Ligament Harvest}

Bovine fibroblasts were isolated from adult bovine and harvested from the metacarpal-phalangeal joints of ligaments obtained from a local slaughterhouse. The ligament was excised from the articulate sac under aseptic conditions, washed with PBS (Phosphate Buffered Saline) ( $\mathrm{pH} 7.4$ ), cut into $1 \mathrm{~cm}$ sections using a sterile scalpel in a petri dish. Then the ligament was digested using $20 \mathrm{ml}$ of Collagenase A (Roche Diagnostics Corporation) in DMEM (Dulbecco's Modified Eagle Medium) culture medium for 36 hours. This enzymatic digestion was performed for 36 hours in petri dish at $37^{\circ}$ and $5 \% \mathrm{CO}_{2}$.

\subsubsection{Bovine Cell Culture}

The mixture was filtered through a $40 \mu \mathrm{m}$ cell strainer (Sigma-Aldrich Inc.) and centrifuged at $700 \mathrm{rpm}$ for 7 minutes. A washing step involved the removal of the supernatant via an aspirator unit and the pellet was resuspended in DMEM with $10 \%$ FBS (Sigma-Aldrich Inc.). This washing step was repeated 2 times. With the suspended cells, Trypan Blue (Invitrogen) was added to determine cellular viability and the total number of cells were determined with a hemocytometer. Cells were seeded at 500000 cells per $75 \mathrm{~cm}^{2} \mathrm{~T} 75$ cell culture flask with $13 \mathrm{ml}$ of DMEM containing $10 \%$ FBS and supplemented with 25 mM HEPES ( $N$-2-hydrocyethylpuperazine- $N$ '-2-ethanesulfonic acid) (Sigma Aldrich Ltd, Oakville, ON), $300 \mathrm{U} / \mathrm{mL}$ penicillin, $300 \mu \mathrm{g} / \mathrm{mL}$ streptomycin, 
and $0.75 \mu \mathrm{g} / \mathrm{mL}$ amphotericin B (Invitrogen Canada Inc, Burlington, ON) (hereinafter referred to as 'standard media'). The cell culture was expanded until $80 \%$ confluency with the standard media being replaced every second day. After $80 \%$ confluent cells were detached from the flasks using trypsin, centrifuged at $700 \mathrm{rpm}$ for 7 minutes, washed twice using the same procedure, and reseeded into new flasks at the initial concentration. Cellular expansion continued until three passages occur (P3) to confirm identity of the cells growing.

\subsubsection{Substrate Seeding of Bovine Fibroblasts}

Cells were gathered from the T75 flasks and centrifuged at $700 \mathrm{rpm}$ for 7 minutes and washed 2 times. The final resuspension was made at a concentration of 100000 cells in $10 \mathrm{ul}$ of standard media. $10 \mu \mathrm{l}$ was seeded onto the substrate creating a tiny bubble over top the micro-post location. The bubble was observed under light microscopy checked every hour until cellular attachment was observed. Following cellular attachment, $3 \mathrm{ml}$ of standard media was pipetted into the well of the 6 well plate by placing the tip on the edge of the well and letting the media rise up over the substrate to prevent cellular detachment. The substrates were cultured for 3 days at $37^{\circ} \mathrm{C}$ and $5 \%$ $\mathrm{CO}_{2}$. The samples will not remain at the bottom of the well due to their low density. Just make sure that the samples are covered at all time by culture media.

\subsection{Post-processing Methods}

Following cell culture multiple processes were conducted to examine the behavior of the bovine fibroblasts on the culture platform. Each process was performed on a separate sample. 


\subsubsection{Scanning Electron Microscopy (SEM)}

To prepare the samples with bovine fibroblasts for SEM imaging they were dehydrated from standard media using a series of graded alcohols. First samples were fixed in 4\% paraformaldehyde (Bioshop) overnight then transfer to $70 \%$ ethanol (ethyl alcohol) for 15 minutes. Remove the $70 \%$ ethanol and replace with $95 \%$ ethanol for 15 minutes. Remove $95 \%$ ethanol and replace with 100\% ethanol (anhydrous) for 10 minutes and repeat the $100 \%$ ethanol change another 2 times. Next, to chemically dry the samples they were grading to $100 \%$ HMDS (hexamethyldisilazane) (Sigma- Aldrich Inc) as follows. A dilution that was 2 parts 100\% ethanol/1 part HMDS was added for 15 minutes. Replace this dilution with a 1 part $100 \%$ ethanol/2 parts HDMS for 15 minutes. Replaced this dilution with $100 \%$ HDMS for 15 minutes and repeat $100 \%$ HDMS exchange one more time. Remove as much of the HDMS as you can and allow the specimen to air-dry in a fume hood (not biosafety cabinet) overnight. Samples were then mounted to appropriate SEM sample mounts using double sided tape. Samples were sputter coated prior to SEM imaging.

Images from the SEM were imported into Image J and the wavelength was measured at 10 separate locations and the average was used to determine the wavelength on the post for the varying flow conditions, see results.

Additionally, the SEM images were used to calculate the number of cells that were bridging between posts, on a single post or on the surface. This was done by manually counting full cell bodies observed in each position for each of the post spacing's an area of $25 \times 25$ posts was analysis. 


\subsubsection{Immunofluorescence staining}

To identify cellular focal adhesions, microfilament locations and the cellular nucleus, immunofluorescence staining was performed. To stain for F-actin, TRITCconjugated Phalloidin (Millipore Cat. No. 90228) $(15 \mu \mathrm{g})$ was reconstituted by suspending into $250 \mu \mathrm{L}$ of methanol. For future use the TRITC-conjugated Phalloidin was aliquot into $50 \mu \mathrm{L}$ into tubes and stored at $-20^{\circ} \mathrm{C}$.

Cells were fixed with $4 \%$ paraformaldehyde in 1x PBS for 15-20 minutes at room temperature. Cells were then washed twice with 1xPBS with 10mM Glycine. Permeabilize cells with $0.1 \%$ Triton $\mathrm{X}-100$ in $1 \mathrm{x}$ PBS for $1-5$ minutes at room temperature. Washed twice with 1 xPBS. Blocking solution of $3 \%$ bovine serum albumin (BSA) in 1xPBS was applied for 60 minutes at room temperature. Dilute the primary antibody (Vinculin Monoclonal Antibody, purified clone 7F9 (Millipore Cat. No. 90227)) to a working concentration of $1 / 500$ in blocking solution of $1 \%$ BSA in PBS and incubate for 1 hour at room temperature. Wash three times (5-10 minutes each) with 1xPBS. Dilute secondary antibody to 1/500 (Gt x Ms, FITC-conjugated, Millipore Cat. No. AP124F) in 1x PBS just before use and incubate for 60 minutes at room temperature. Double labeling was performed during the secondary antibody stain by diluting TRITC conjugated Phalloidin to 1/1000. Wash was performed three times (5-10 minutes each) with 1x PBS. Following the washing step, nuclei counterstaining was performed by incubating cells with DAPI (Millipore Cat. No. 90229) at a dilution of 1/1000 for 5 minutes at room temperature, followed by washing cells three times (5-10 minutes each) with 1xPBS. Cells were mounted on a coverslip using antifade mounting solution 
(Vectashield mounting medium, $\mathrm{H}-1200$ ) and visualized with a Confocal Zeiss LMS700 with ZEN Black acquisition software. Reconstruction in Imaris.

\subsubsection{Fluorescence Recovery After Photobleaching (FRAP)}

FRAP was used to analyse three separate experiments. First, native bovine ligament was examined to observe the response of native tissues to FRAP protocols. The bovine ligament was extracted and cut into slices as thin a possible and placed into a 6 well plate. A solution of $0.2 \mathrm{M}$ Calcin AM (Molecular Probes Viability/Cytotoxicity Kit, ThermoFisher) was mixed in PBS and added to the well plate. The well plate was incubated with Calcin solution for 20 minutes at $37^{\circ} \mathrm{C}$. The Calcin solution was removed and the ligament was washed with PBS two times. A drop of standard media was placed on a glass cover slip and then the ligament was added into the drop. A second cover slip was placed over top and visualized with a Confocal Zeiss LMS700 with ZEN Black acquisition software with the FRAP add-on. Image time series were taken every 30 seconds and for the photobleaching, 20 iterations using $488 \mathrm{~nm}, 10 \mathrm{~mW}$, class 3B laser at $100 \%$ power was performed.

Similarly, the second experiment used previously extracted and passaged fibroblast cells which were already seeded onto the micropost platform. The same protocol was performed on the platform experiments as above.

The third experiment aimed to remove the communication between bovine fibroblasts on the micropost platform. To block the gap channels $500 \mu \mathrm{M}$ solution of GAP27 (Tocris Cat. \#1476) was preincubated with the bovine fibroblasts for 3 hours before seeding the cells onto the micropost platform [94]. Following the 3 day cell culture the same protocol was performed as stated above. 


\section{Results and Discussion}

Many methods exist to create wrinkles, in recent years, planar surfaces have been able to achieve nanowrinkles on planar surfaces [59], [71], [95], [96]. Huntington et. al. created a tunable platform that fabricates nanowrinkles from $30 \mathrm{~nm}$ to $500 \mathrm{~nm}$ and Liam et. al. produced nanowrinkles $85 \mathrm{~nm}$ to 4 um [71], [95]. However, the fabrication of wrinkles on a non-planar surfaces remains limited. Three methods were tested to achieve a tunable platform for nonplanar nanowrinkles.

\subsection{Nonplanar Cell Culture Environment to promote cellular network formation}

After the fabrication of the posts using photolithography three washing methods were tested to create a reliable tunable control for wrinkled morphology on the posts.

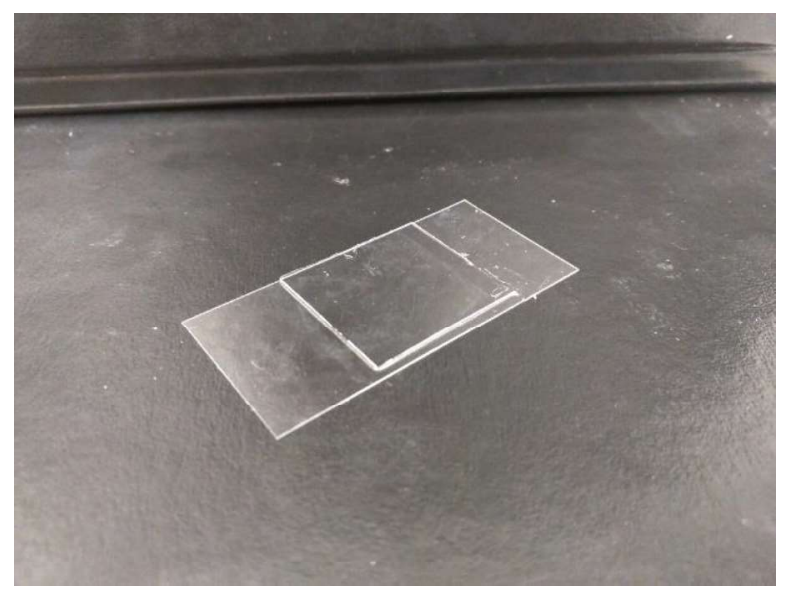

Figure 4.1: Substrate, substrate with posts before washing

The fabrication method leaves behind excess PEDGA solution which needs to be washed away leaving an even layer of PEG-DA solution only on the posts. The amount of PEG-DA solution remaining determines the wavelength of the wrinkles created following treatment with plasma [54]. 


\subsubsection{Diffusion method}

The first washing method tested was simple diffusion. Following post fabrication, the substrates were placed in a petri dish filled with $70 \%$ ethanol and allowed to soak for two hours, setup and results shown in Figure 4.2a.

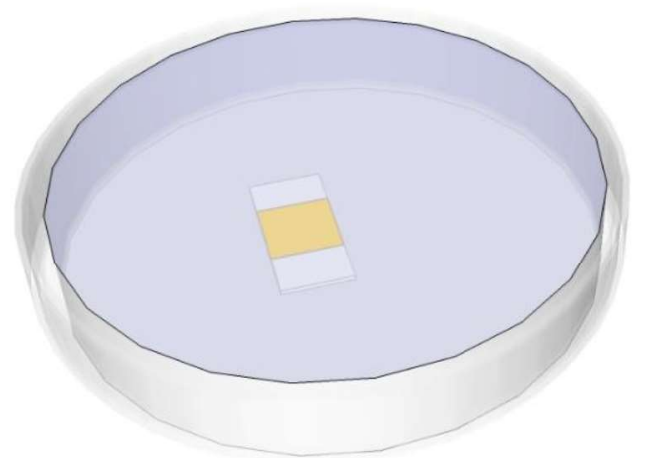

A

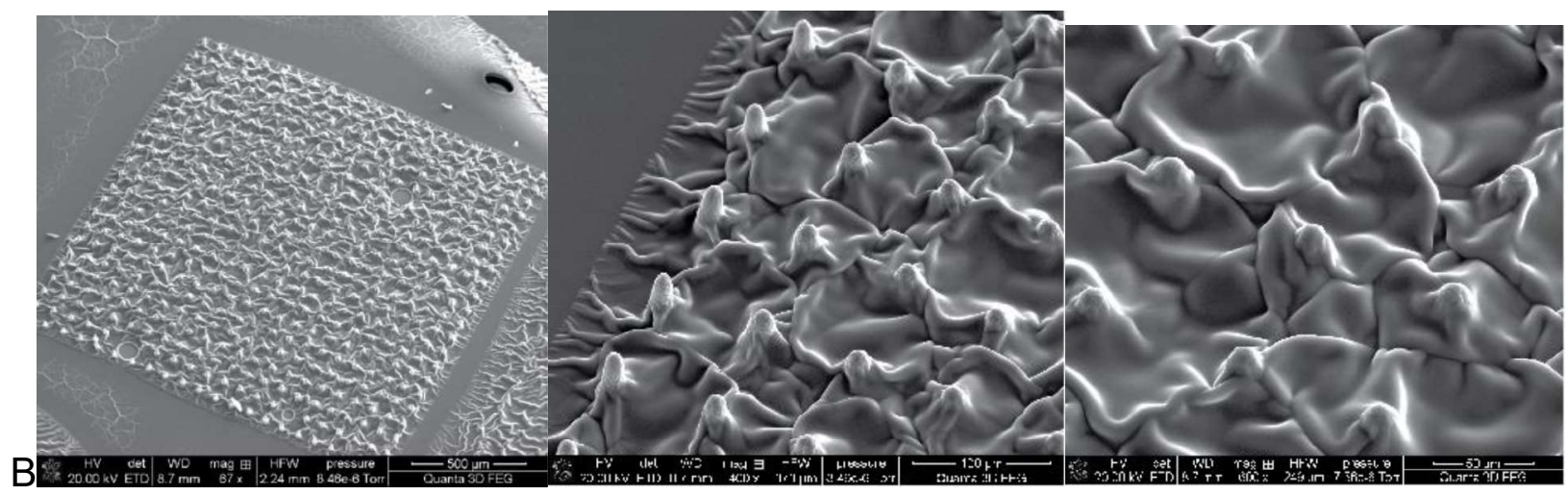

Figure 4.2: (A) Diffusion method setup; (B) Results from diffusion method

The pure diffusion platform resulted in a substrate that were hard to distinguish between the posts and the bottom surface, Figure $4.2 \mathrm{~b}$. The ethanol was unable to penetrate in-between the posts and created a completely covered base layer of wrinkles. Additionally, a large difference was observed between the outer posts and the inner posts on the square. As expected with diffusion, the outer posts exhibited a 
smooth topography while the inner posts were still completely covered. Overall, this washing method proved ineffective, uneven, and took a long time.

\subsubsection{Droplet method}

To increase effectiveness and conformity of all wrinkles on the posts during the washing process a droplet method was tested. Initially, holding the glass substrate on approximately a $45^{\circ}$ angle, $500 \mu \mathrm{l}$ of $70 \%$ ethanol was dripped over the posts, Figure 4.3a.

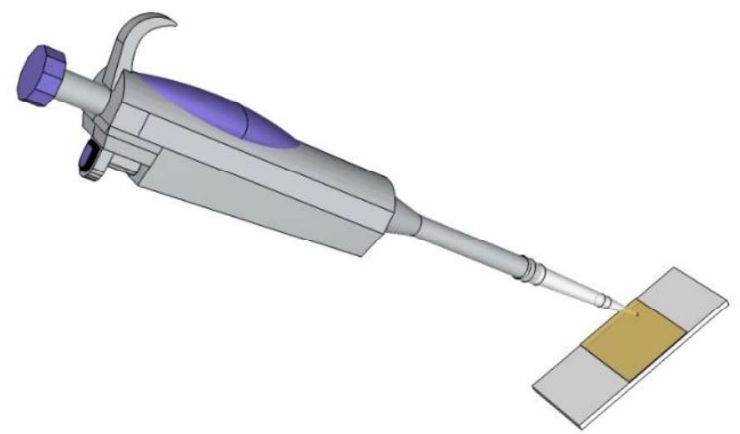

A

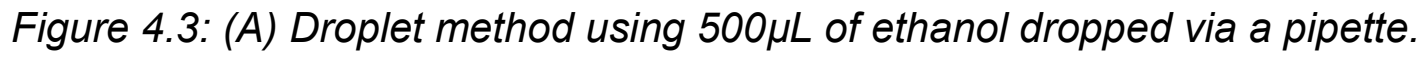

However, to make this setup more consistent, modifications to make this method more quantitative were made. The second setup for the droplet method consisted of fabricating a glass cover slip holder that maintained a constant $45^{\circ}$ angle and a burette placed $4 \mathrm{~cm}$ above the working surface. A notch was placed on the valve of the burette 
as to allow for opening to the same location every time to provide consistent drop size, Figure 4.3b.

This fabrication method proved to provide good post shape with defined wrinkles, Figure 4.4. However, the ability to fabricate a desired wavelength repeatedly was difficult. This can be observed in Figure 4.4, where multiple results using exactly two drops demonstrate varied from nanoscale wrinkles to microscale wrinkles.
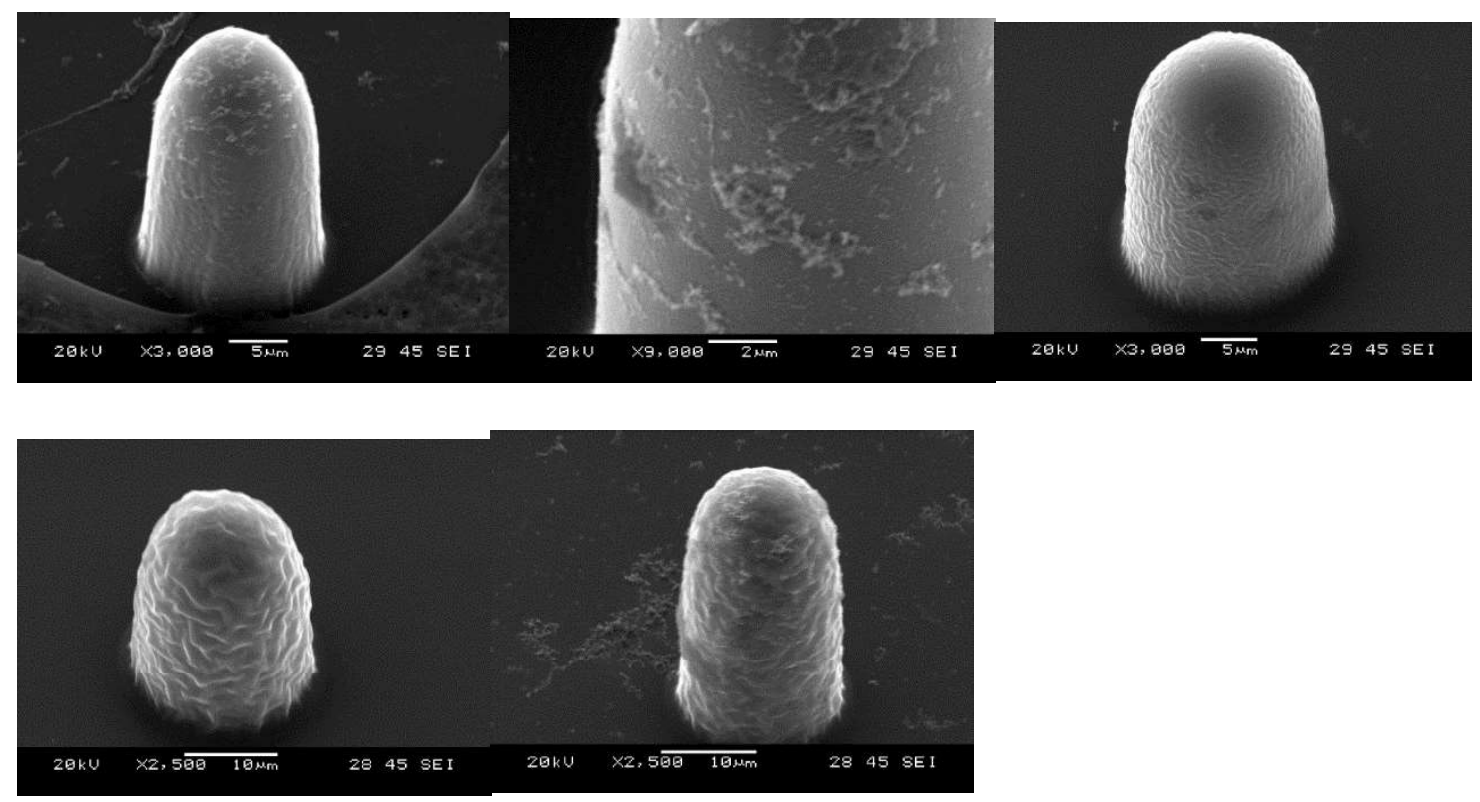

Figure 4.4: Characterization of Drop Method. All with 2 drops.

\subsubsection{Microfluidic method}

Creating a more tunable platform system was desired to produce controllable wavelengths, thus, a more precise washing method was developed which allowed greater control over the amount of time ethanol flowed over the posts and the flow rate of the ethanol. A microfluidic pump with a $10 \mathrm{ml}$ syringe of $100 \%$ (anhydrous) ethanol 
and tubing with a 16-gauge blunt point needle inserted into the PDMS microchannel was used to control these variables, Figure 4.5.

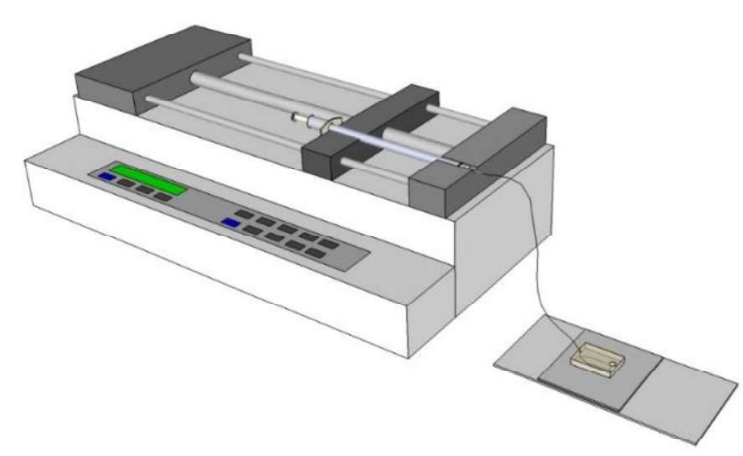

Figure 4.5: Microfluidic method setup.

Using the microfluidic pump in infuse only mode, the settings were set to $10 \mathrm{ml}$ syringe with the desired flow rate (between 50 and $200 \mu \mathrm{L} /$ minute). The plunge was advanced until ethanol exited the end of the needle to prevent air from entering the microchannel. Then the needle was inserted into channel inlet of the microchannel and the microfluidic pump was started for the desired amount of time. Make sure steady state has been achieved for the ethanol flow so no additional pressure is backed up from advancing the platform which would cause an increased flow rate or extended time delay to stop. 

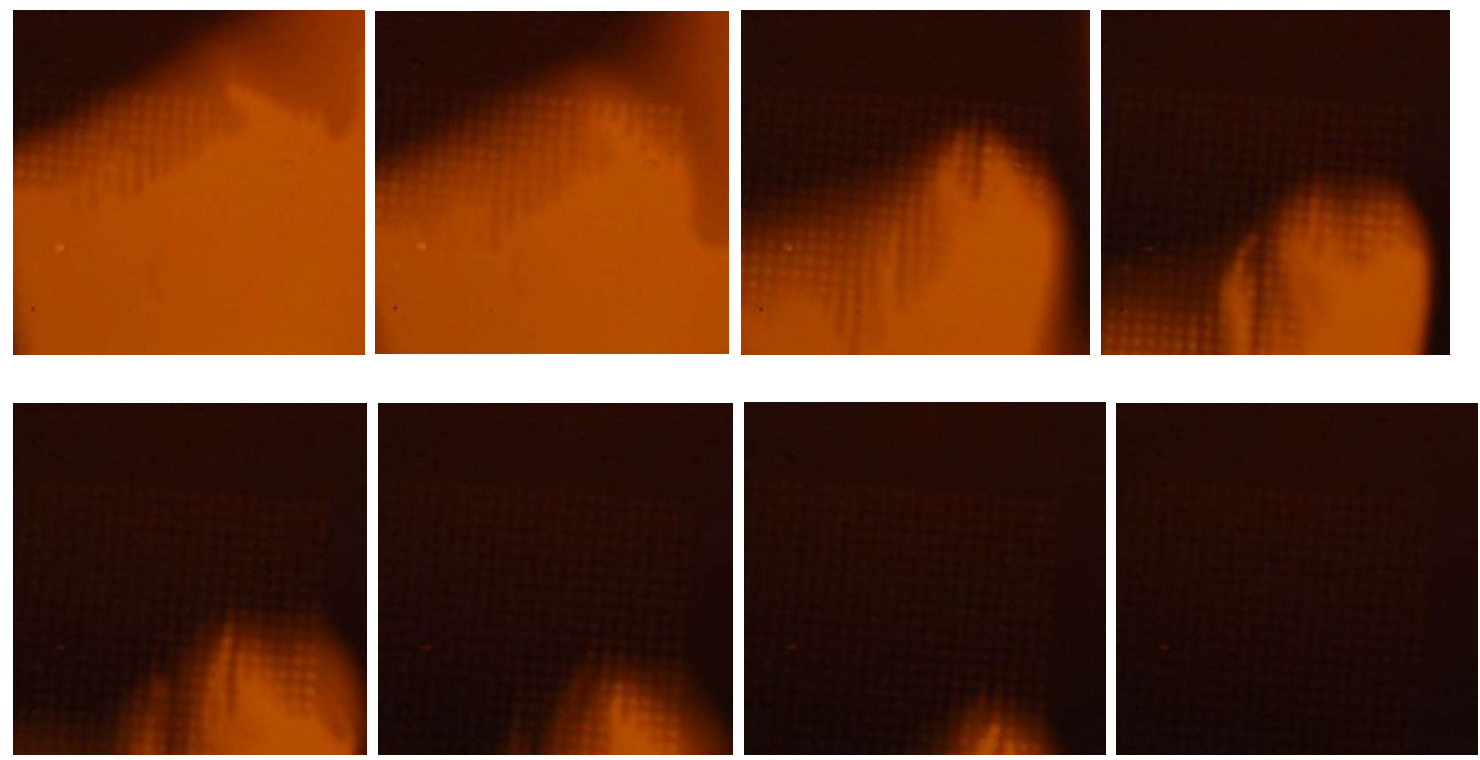

Figure 4.6: Visualization time capture of microfluidic post washing method using Rod-B with fluorescent video capture.

The exact removal of PEG-DA varied due to the velocity profile of ethanol. In addition, the direction of flow could change slightly depending on needle position in the inlet. This method was visualized by the addition of Rodamina-B and fluorescence imaging with a Zeiss AxioObserver, Figure 4.6. The microfluidic post washing method produced repeatable wrinkle morphology, Figure 4.7.
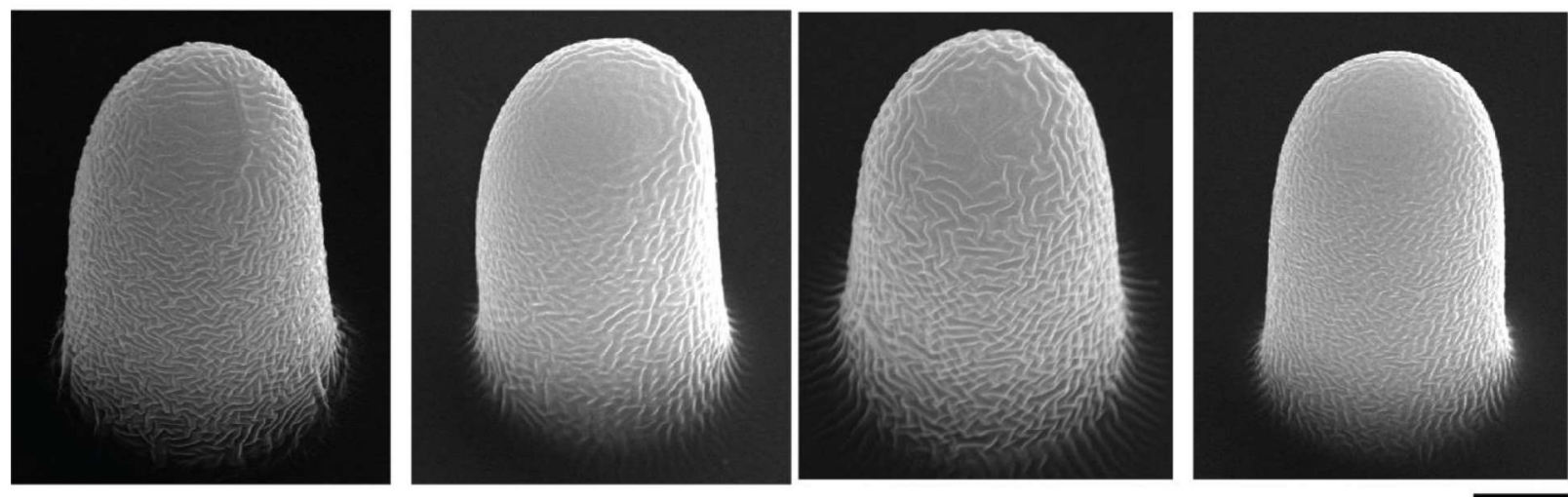

Figure 4.7: Characterized microfluidic method, posts have repeatable tunable wrinkle morphology. Scale bar $5 \mu$ m. 
Bhawalkar et al's colloidal lithography method for patterning nonplanar surfaces creates a multiple hierarchy with the use of both nanoscale and microscale nonplanar structures [61]. However, the mechanism used is non-selective and covers both the surface and the nonplanar structure Figure 4.8a. The nonplanar surfaces created via photolithography and the microfluidic washing method provides selective microscale and nanoscale patterns on a designable nonplanar structure, Figure $4.8 \mathrm{~b}$. The surface on this platform has to potential to be modified through additional methods applied either prior or post wrinkle formation. Such methods include being tagged with cell evading proteins, different cellular attraction methods/cell capturing, etc. [11], [97]-[99].
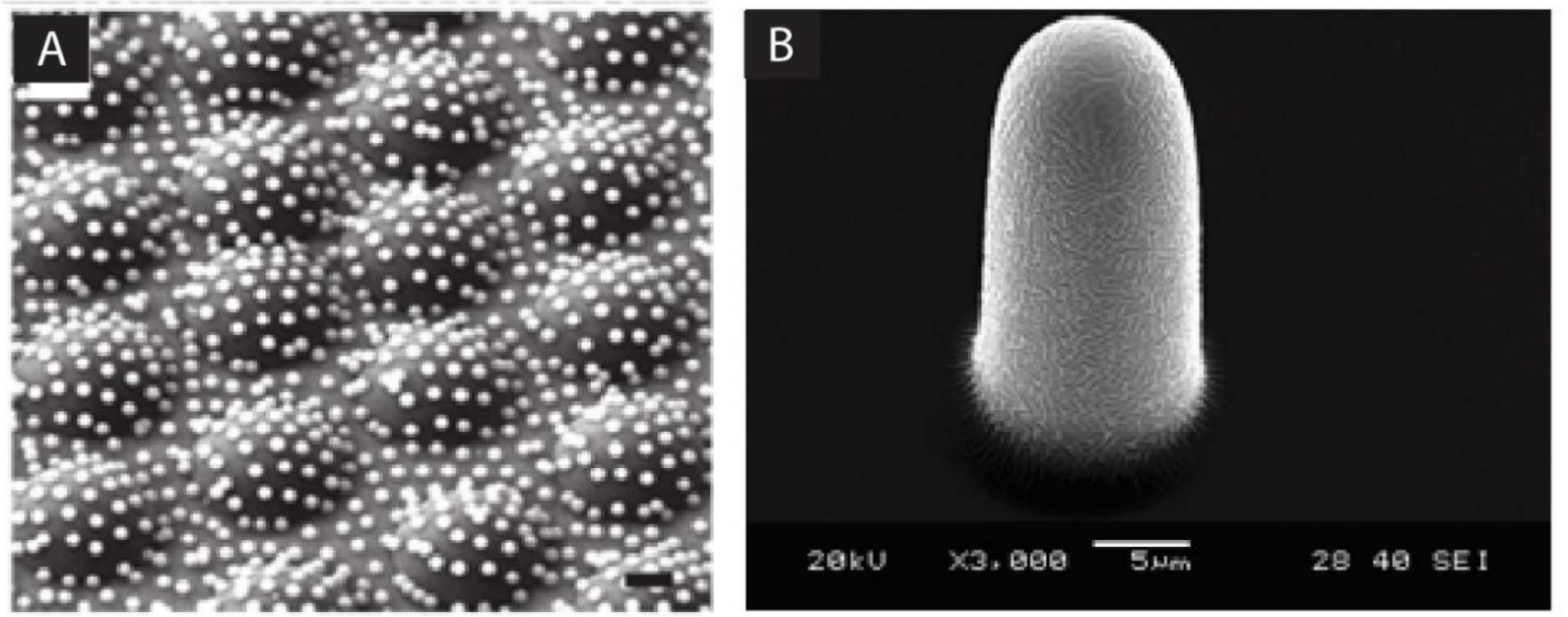

Figure 4.8: Comparison of nonselective vs selective nano-pattern. A) nanoscaled geometry appears uniformly across entire surface [61] B) wrinkles only appear on the nonplanar geometry.

Micromolding has demonstrated the potential to achieve structures that are both hierarchical structures and has the potential to incorporate wrinkles [75]. However, the speed or fabrication of these platforms remains a challenge [75]. In addition, the models created are designed manually and not naturally occurring [75]. The method presented achieves this desired wrinkled hierarchy in a rapid one step method. 


\subsection{Substrate Characterization}

All results hereon in are processed using the microfluidic post washing method.

\subsubsection{Focus depth and UV Intensity}

The effect of change in focus of the microscope on disappearance of wrinkles from the base substrate and formation shape was found to be a significant factor. The initial focus (at the thinnest achievable line) resulted in a completely covered surface base with wrinkles as a result of out of focus UV polymerization, Figure 4.9 at $0 \mu \mathrm{m}$. This was due to a transition which occurs as the focal plane (red) in lowered from the top of the microchannel down, Figure 4.9.

The transition observed was a result of a combination of the gradient of the UV light and the focal distance [54], [56]. At $0 \mu \mathrm{m}$ focal distance down, the UV light polymerizes the entire surface (blue) causing a full surface wrinkling during plasma treatment. At $\sim 10 \mu \mathrm{m}$ focal distance down, a dispersed pattern of the photo mask can be observed at the base where the UV light is the strongest. At $\sim 20 \mu \mathrm{m}$ focal distance down, full post height is achieved with no dispersed pattern. Past $\sim 30 \mu \mathrm{m}$ the post height shortens due to a reduced UV strength. Thus, the optimum focus depth was determined to be $\sim 20 \mu \mathrm{m}$. Past the center focused line, the UV intensity is decreased significantly and results in a reduced post height but no polymerization from out of focused light, Figure 4.9 . 

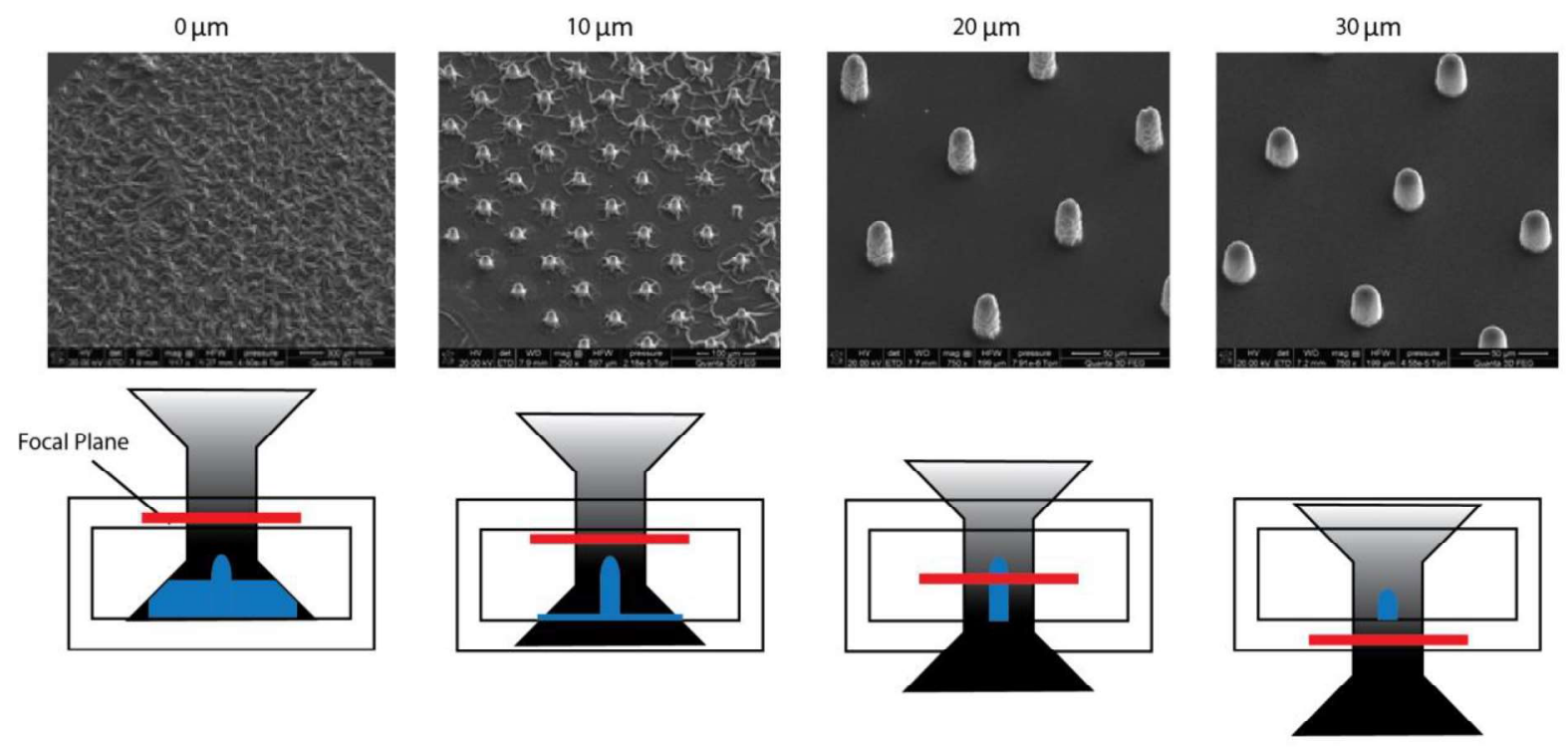

Figure 4.9: Summary of focusing effect in channel on post structure.

\subsubsection{Tuneable wrinkle wavelength platform}

Using the microfluidic pump method, the thickness of the partially polymerized monomer layer remaining on the surface was adjusted, Figure 26 . To create nanoscale wrinkles the washing time was increased from 15 to 120 seconds. However, using only one washing time did not alter the wavelength of the wrinkles significantly. Thus, adjusting 


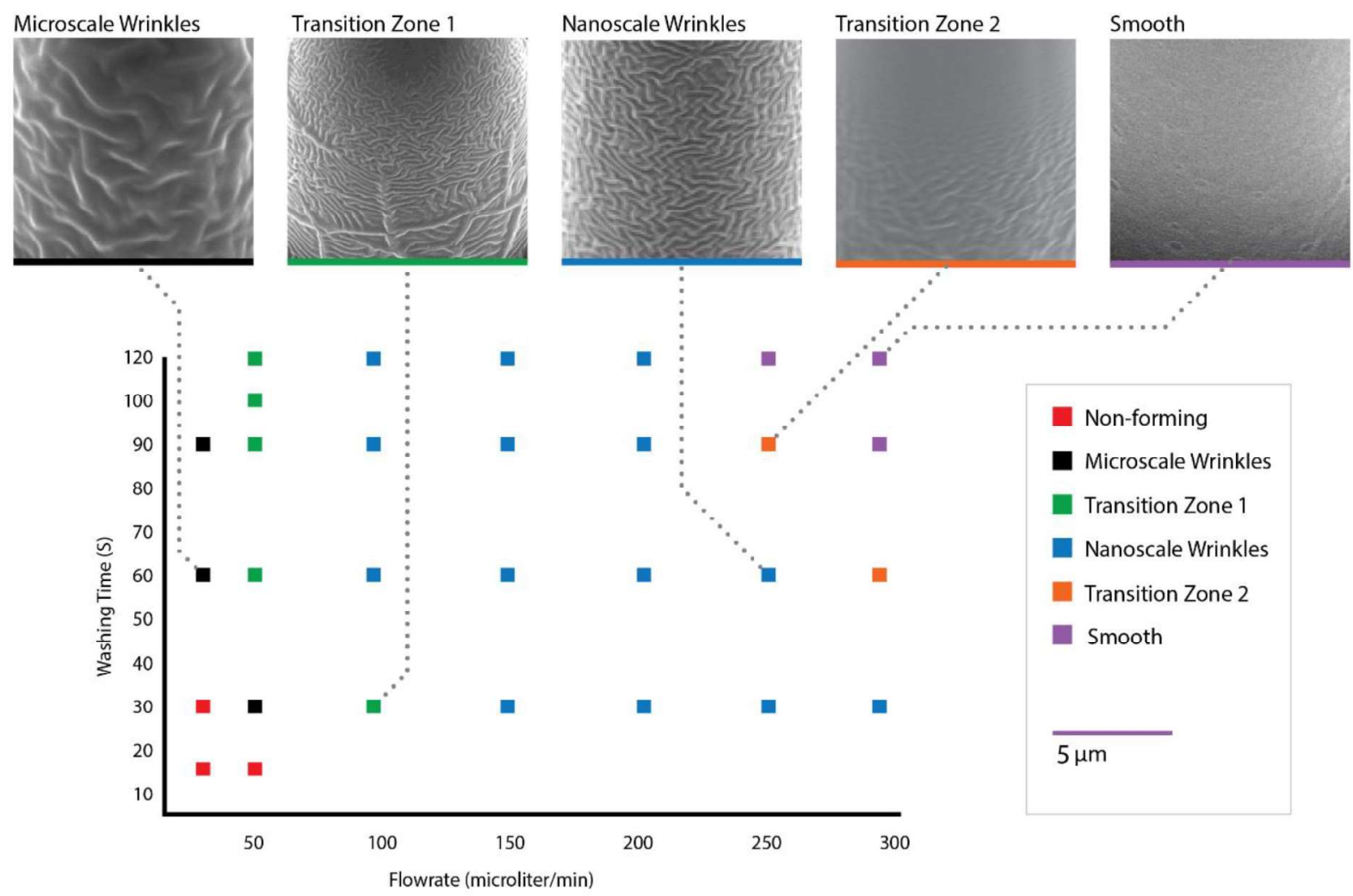

Figure 4.10: Characterization of microfluidic washing to achieve a tuneable platform via washing time or flowrate.

the flow rate from 50 to $300 \mathrm{ml} / \mathrm{min}$ was also analysis. With the higher flow rates, multiple transitions were observed. At $20-50 \mathrm{ml} / \mathrm{min}$ microscale wrinkles were formed. This was similar to results observed under similar conditions without an adjustment to control the process [54]. At 50-100 $\mathrm{ml} / \mathrm{min}$ a transition zone occurred, due to the velocity profile in a microchannel. A similar transition zone occurs between microscale and nanoscale wrinkle morphology and between nanoscale and smooth wrinkle morphology. The transition structures contain bi-regional wrinkles Figure 4.11. Figure 4.11a, shows the transition zone between microscale and nanoscale wrinkled structures with the nanoscale region increasing with increase in washing time and flow rate. Figure 4.11b, shows the transition zone between nanoscale and almost smooth structures with smooth region increasing with increases in washing time and flow rate. 

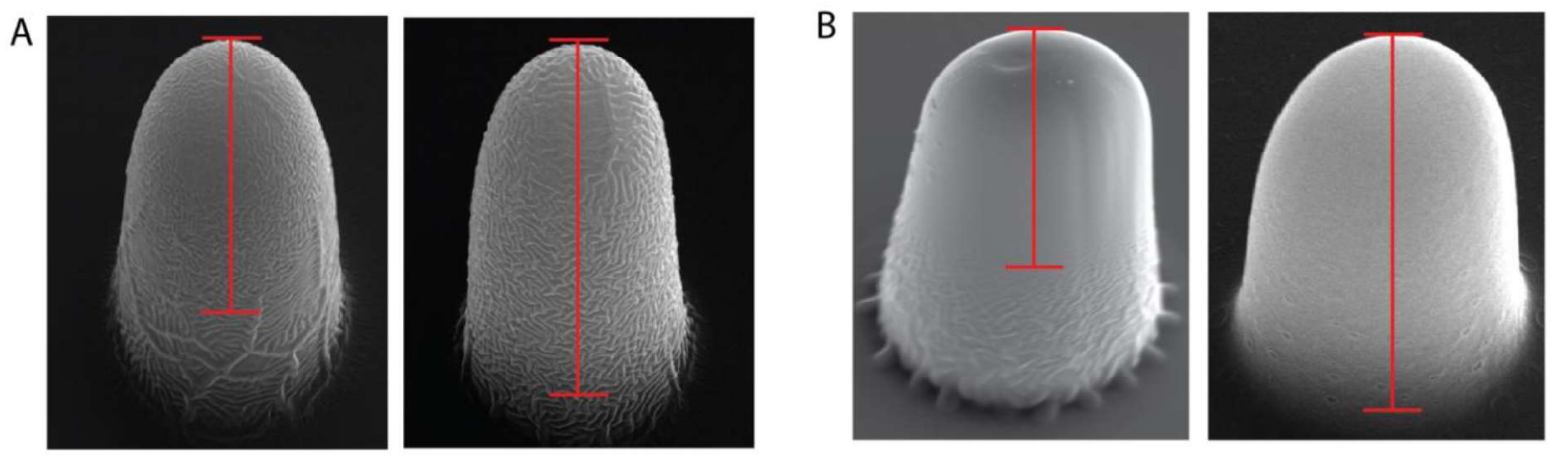

Figure 4.11: Transition zones containing bio-regional wrinkles $(A)$ micro to nano transition (B) nano to smooth transition

Adjusting the flowrate between $100-250 \mathrm{ml} / \mathrm{min}$ resulted in almost uniformity nanoscaled wrinkled structures. Thus, the flow rate was determined to be more effective than washing time to precisely adjust the thickness of the polymer remaining. Using the microfluidic pump method provided precise and accurate control over the wrinkle morphology with a wide range of flow conditions available to produce nanowrinkles. Smooth morphology was achieved only at the high ends of both washing times and flow rates.

\subsection{Cellular Response to Post Topography}

\subsubsection{Wrinkled Topography Cell Response}

Significant preference was observed by the bovine fibroblasts for posts with any wavelength of wrinkles over smooth posts, Figure 4.12. Wrinkled posts were engulfed by a cell that came in contact with the post, however, cells did not differentiate smooth posts to be more advantageous than the surface and did not climb over the posts. 


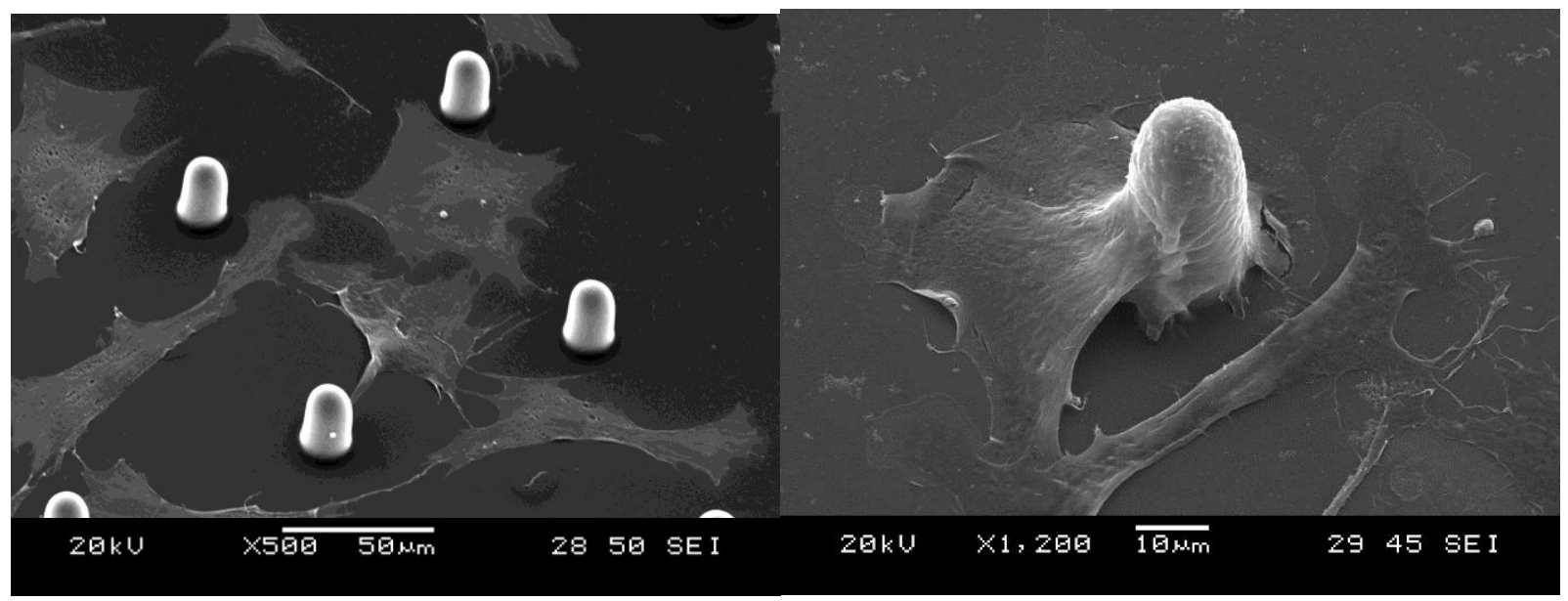

Figure 4.12: Cell experiments show cells ignore smooth posts but prefer to attach to wrinkled posts (A) SEM images show cells spread on substrate and ignore the smooth posts. (B) A representative SEM image shows that bovine fibroblasts attach to wrinkled posts after 3 days' cell culture and they climb onto the posts and conform to them, forming cell wrapped posts.

\subsubsection{Critical Bridging Distance}

Spacing between the posts was altered from 50 um to 110 um of the posts to determine if there was an optimum distance for bovine fibroblasts. At 110 um spacing, no bridging was observed. However, cells do maintain preference for wrinkled post morphology and a high number of single cells are engulfed on one post. At $90 \mu \mathrm{m}$ spacing, a bridging phenomenon was observed occasionally between vertical/horizontal posts but no bridging was observed on diagonals. For 70um spacing bridging occurred in the vertical/horizontal mainly however, a few bridges do occur in the diagonal. at a much less frequency than both 50 and $70 \mu \mathrm{m}$. At $50 \mu \mathrm{m}$ spacing, bridging was observed in all directions, both diagonal and vertical/horizontal. Long chains of overlapping cellular bridging formed on a grid of posts at $50 \mu \mathrm{m}$ spacing between adjacent posts. 


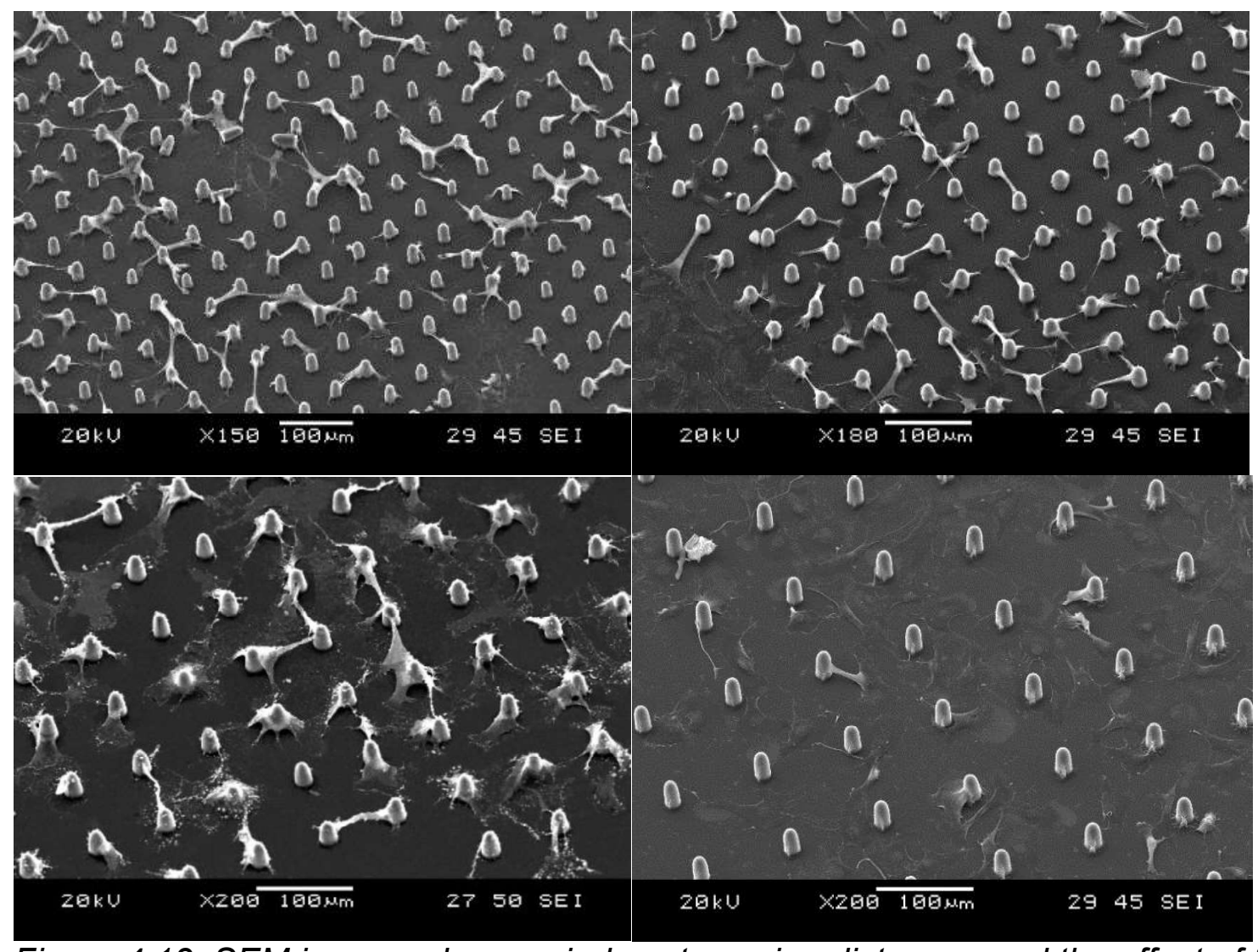

Figure 4.13: SEM images show varied post spacing distances and the effect of the

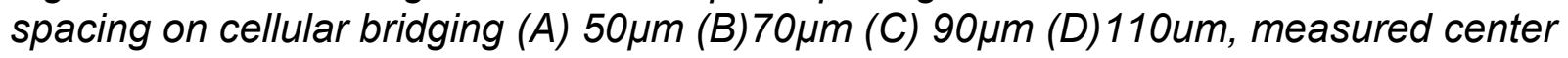
to center 
These trends can be observed in the graph below, Figure 4.14.

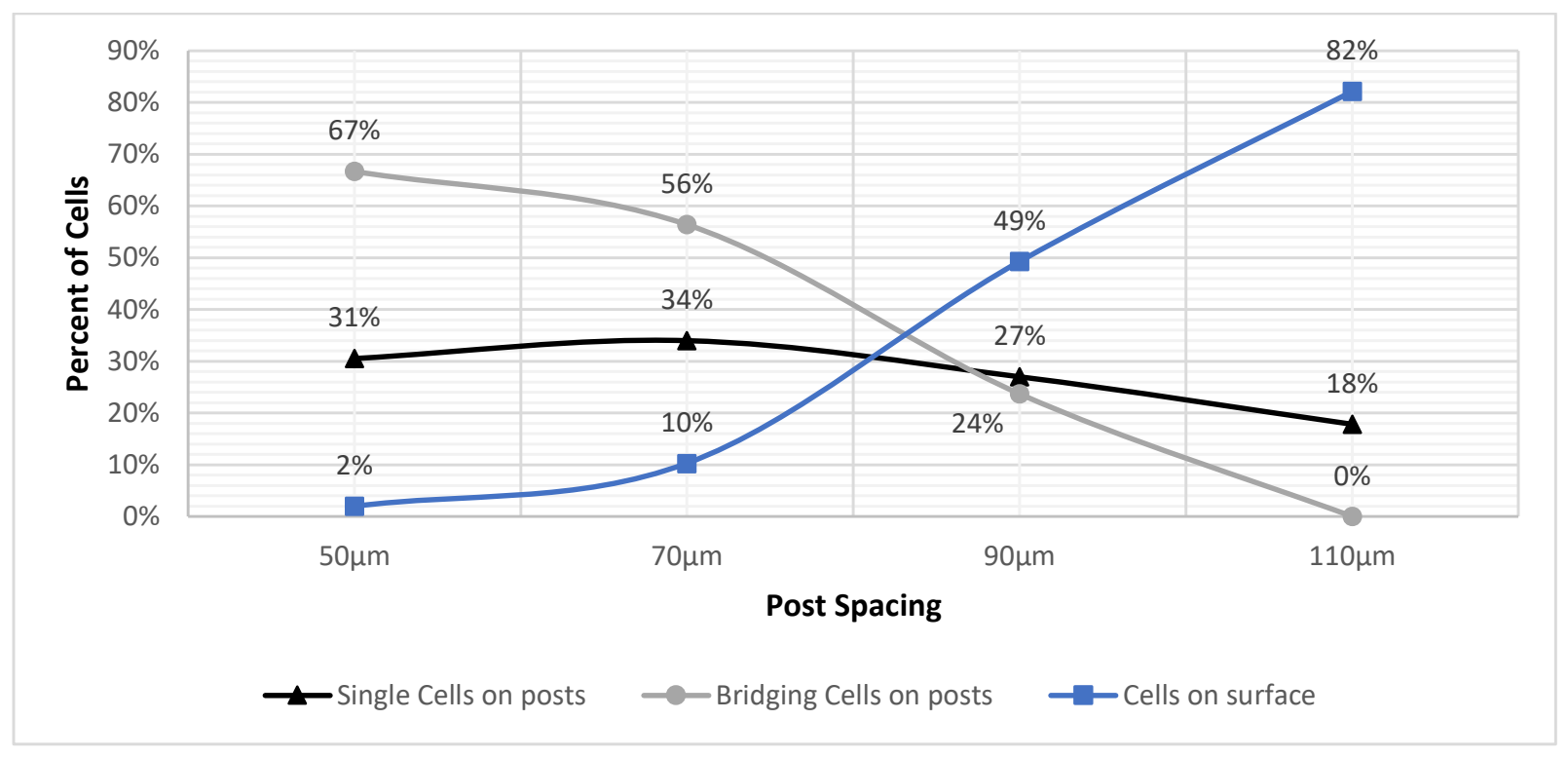

Figure 4.14: Trends for post spacing and cellular attachment

Previous studies pointed to cellular preference attachment on wrinkled surfaces and alignment of cellular structures [59], [76], [78]. Significant preference was observed by the bovine fibroblasts for posts with wrinkles over smooth posts, Figure 4.12.

Wrinkled posts were engulfed by a cell that came in contact with the post, however, cells did not differentiate smooth posts to be more advantageous than the surface and did not climb over the posts. Cellular preference for wrinkled surfaces demonstrates a geometry induced thigmotaxis.

During this study, bovine fibroblasts established the ability to bridge between two posts promoting the exploration of a critical bridging distance. As the post spacing increased, cell bridging between posts decreased. Similarly, the cells remain on the surface at a higher degree on the surfaces with wider spacing even though the chemical composition of the surface was not altered. Overall, the percentage of cells that climb and maintain morphology on a single post remains relatively constant, not dependent of 
the post spacing. Thus, for bovine fibroblasts the maximum stretching distance over a single gap we find is $90 \mathrm{um}$. Levina et. al. proposed the maximum spreading length of fibroblasts increases with time, Figure $2.9 \mathrm{~g}$, and determined the maximum length to be approximately $150 \mu \mathrm{m}$ with the use of confinement [77]. This differs from the distance observed in our platform due to Levina et al.'s use of a planar surface allowing the cell continuous contact guidance. According to Levina, the maximum length was observed to occur approximately 24 hours after fibroblasts seeding and determined that fibroblasts adopt the same length [77]. Thus, our 72-hour culture exceeds this time frame and all fibroblasts experience their maximum length. Another study reported a maximum distance of $25 \mu \mathrm{m}$ after this point, fibroblast spreading was limited and cells became triangular, ellipsoid or round [100].

On a surface of wrinkles, from $2 \mu \mathrm{m}-30 \mu \mathrm{m}$ wavelength range, fibroblasts elongated and aligned when compared to flat surfaces [76]. Additionally, the wrinkled surfaces resulted in an increase of unipolar fibroblasts, whereas, on the flat surfaces fibroblasts are observed to be multipolar, Figure 4.15a,b [76]. A similar observation occurred with the designed wrinkled micropost platform designed. On bridging fibroblasts, lower inter-post spacing platforms, the fibroblasts maintain the same unipolar morphology, Figure 4.15c. However, on the high inter-post spacing, fibroblasts that engulfed only a single post tended to show a more multipolar cell morphology, Figure 4.15d. 

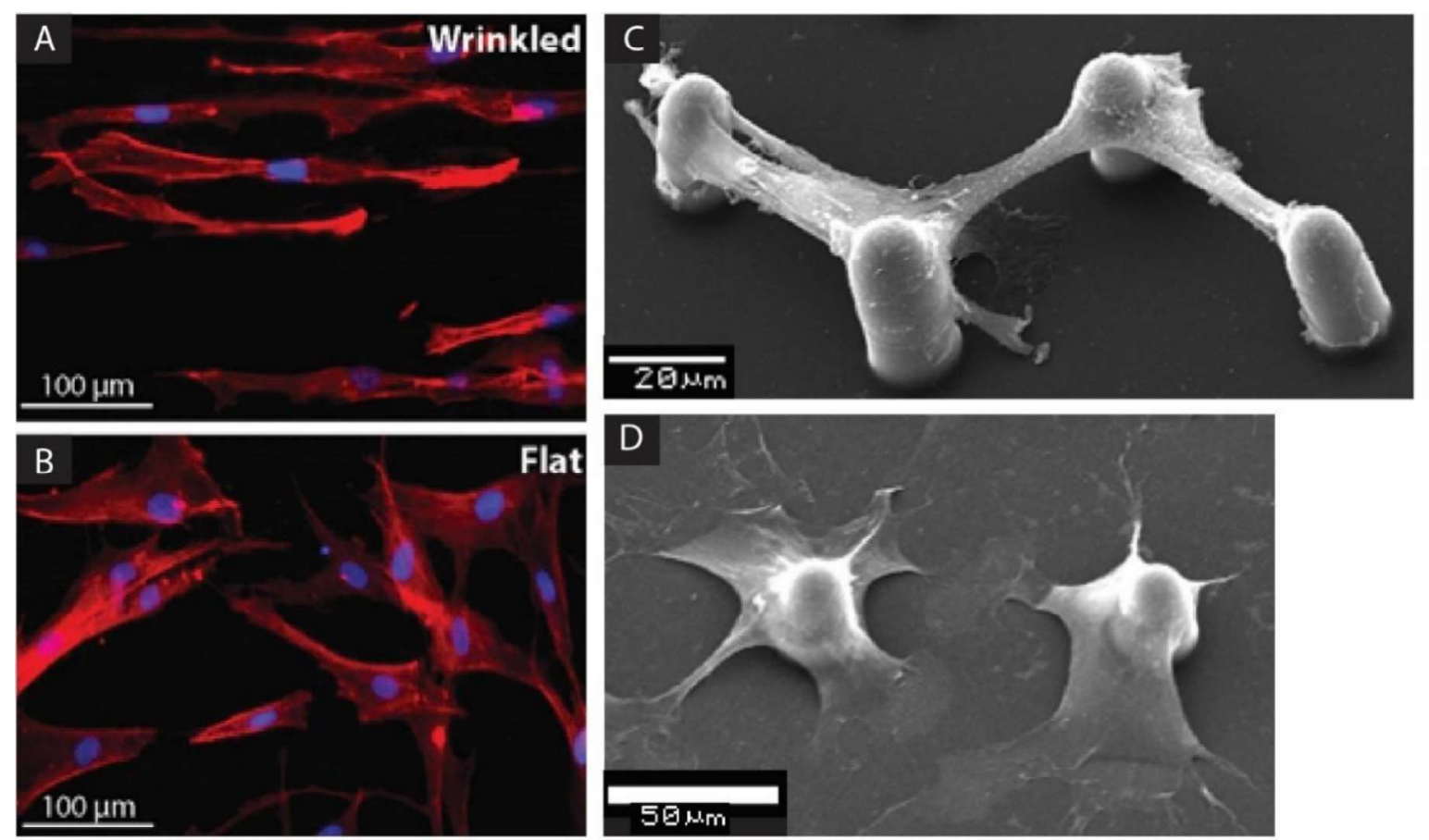

Figure 4.15: Polarity of fibroblasts A)microscale wrinkled planar surface maintains unipolar fibroblasts [76] B) flat planar surface presents multipolar fibroblasts [76] C) bridging fibroblasts maintain unipolar morphology D) non-bridging fibroblasts demonstrate a multipolar morphology.

As outlined in the literature review, directing of growth via many types of topographical features is possible. Applications for microposts in literature have mostly been applied to measure contractile forces within individual cells and effects of substrate stiffness [35], [101]. Typically, these micropost arrays have an addition of an attractor to the top of the micropost, such as fibronectin, to enhance cellular movement onto the posts [35]. However, due to the preference of wrinkles being present on the fabricated microposts, addition of proteins was not necessary in our model. Bovine fibroblasts attached to the microposts with focal adhesion complexes, as determined by the color of yellow in the immunostaining confocal images Figure $4.16 a$ and b, $50 \mu m$ and $70 \mu \mathrm{m}$ respectively. The complexes are present at the site of both F-actin and vinculin. The location of these focal adhesions complexes are much more concentrated than when the cell is spread out on the surface Figure $4.16 \mathrm{~d}$. It is noticeable that the 
presence of $\mathrm{F}$ - actin is in a higher degree at the connection of the post into the cellular bridge where the highest degree of tension will occur.

Examination of higher inter-post spacing samples sometimes resulted in cells which connected in the middle of the two posts, as opposed to overlapping on the posts themselves, Figure 4.16c. This connection contained focal adhesion complexes, not adhered to the substrate surface, but to the other cell. Many biological tissues require the alignment and organization of cells to form, such as ligaments and neurons.
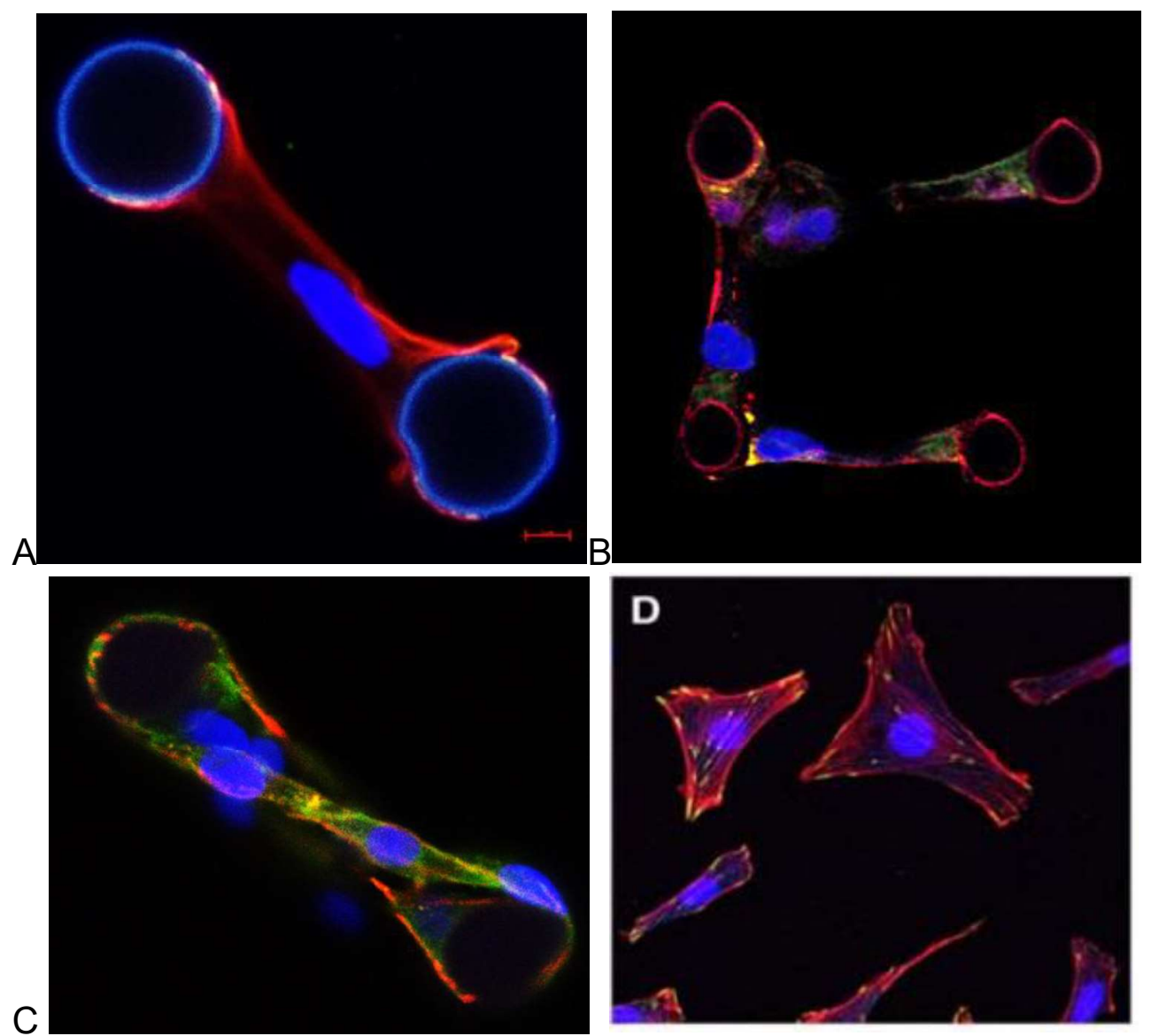

Figure 4.16: Immunostaining of bovine fibroblasts bridging posts vs on a planar surface. Staining for actin vinculin nucleun phallodin. The focal adhesion complexes can be observed at random points on the planar surface. However on the microposts they are present only on the post.

Similar morphology has been demonstrated for fibroblasts using ECM proteins, fibronectin and vitronectin, microprinted onto the surface [100]. Potential exists to 
design a similar communication network. However, these provide a 2D analysis. The network developed on the microposts provides the cell a 3D environment which more accurately represent true physiological cells.
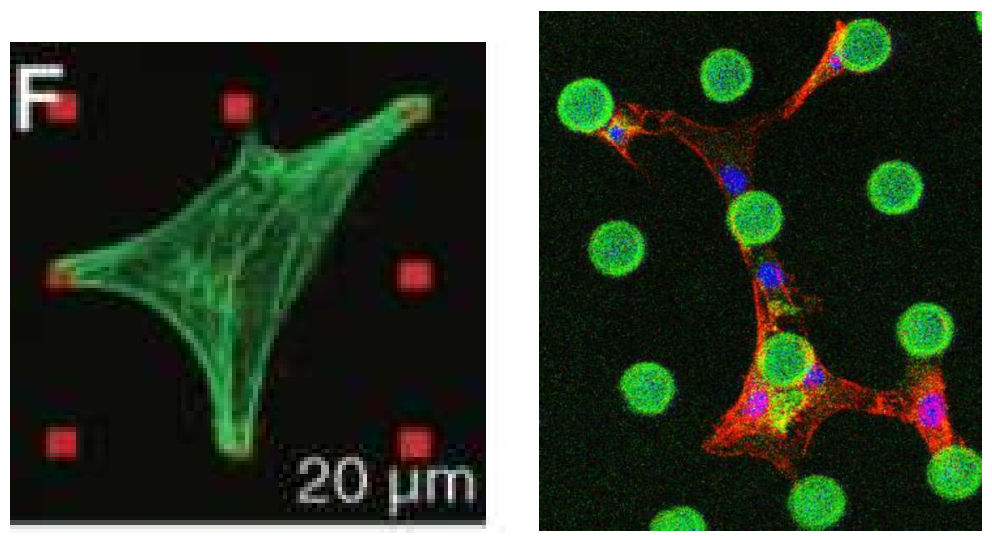

Figure 4.17: cells spread actin cytoskeleton stained formed stress fibres between dots, dots of microcontact printing of ECM protein, B16 cells - mouse melanoma cells. gold coated coverslip, inked with hydrophoblic alkanethiol . [100]. At a distance of $25 \mu \mathrm{m}$, spreading was limitedand cells became triangular, ellipsoid or round. Post spacing of 50um.

\subsubsection{Communication - FRAP}

These biological tissues which rely on alignment of cells depend heavily on cellcell communication. By combining nanoscale wrinkled microposts with a cellular communication an environment to enhance cellular network formation was developed. Current methods of cellular communication are missing the ability to control the cells while still maintaining communication [5], [102].

To investigate cellular communication existed between bovine fibroblasts on this platform, FRAP was performed under three scenarios, Figure 4.18. The first, was on a native ligament immediately following dissection. The second was on extracted bovine fibroblasts on the platform, and the third used a gap junction inhibitor, GAP27. Comparing the kinetics of the three experiments, the recover observed in the native ligament was similar to the recover observed on the platform without GAP27. GAP27 
was added to see if communication could be blocked from occurring. The GAP27 inhibitor decreased the recovery response, indicating a simple block of the gap junctions. However, this slight recovery may continue after 700 seconds due to additional methods of entry into the cell such as another demonstrated cx36 as suggested by Yang et al [103]. The response observed in fibroblasts during FRAP matches other responses observed in literature [85], [86].

The kinetics were detected using the built in FRAP analyser on the ZENN Black software. The communication detected in native bovine ligament was comparative to the response of the fibroblasts on the bridging posts Figure 4.19. 


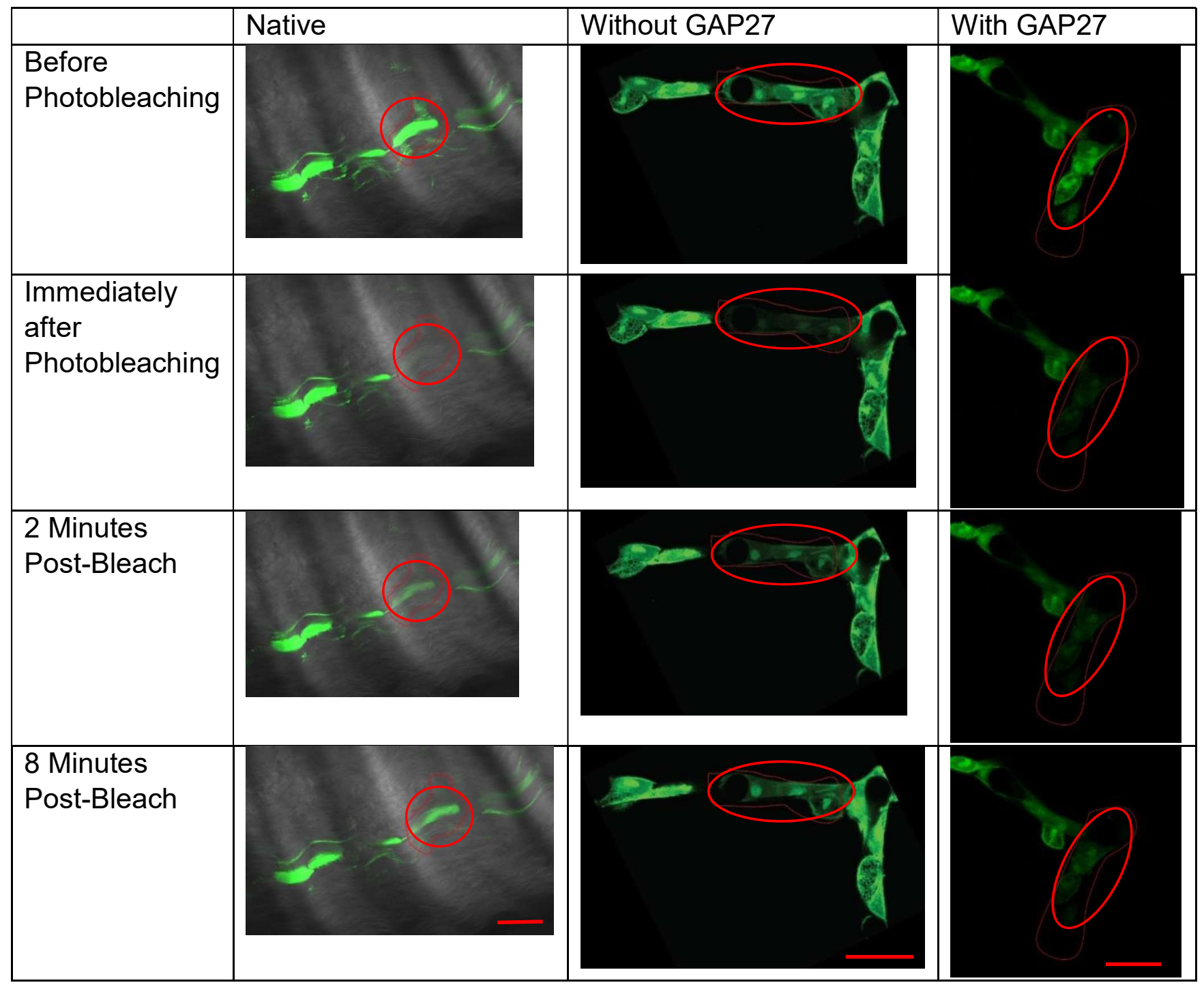

Figure 4.18: FRAP (Fluorescence recovery after photobleaching) demonstrates basic communication exists scale bar $=25 \mathrm{um}$. Red circles is areas where laser was applied for photobleaching 


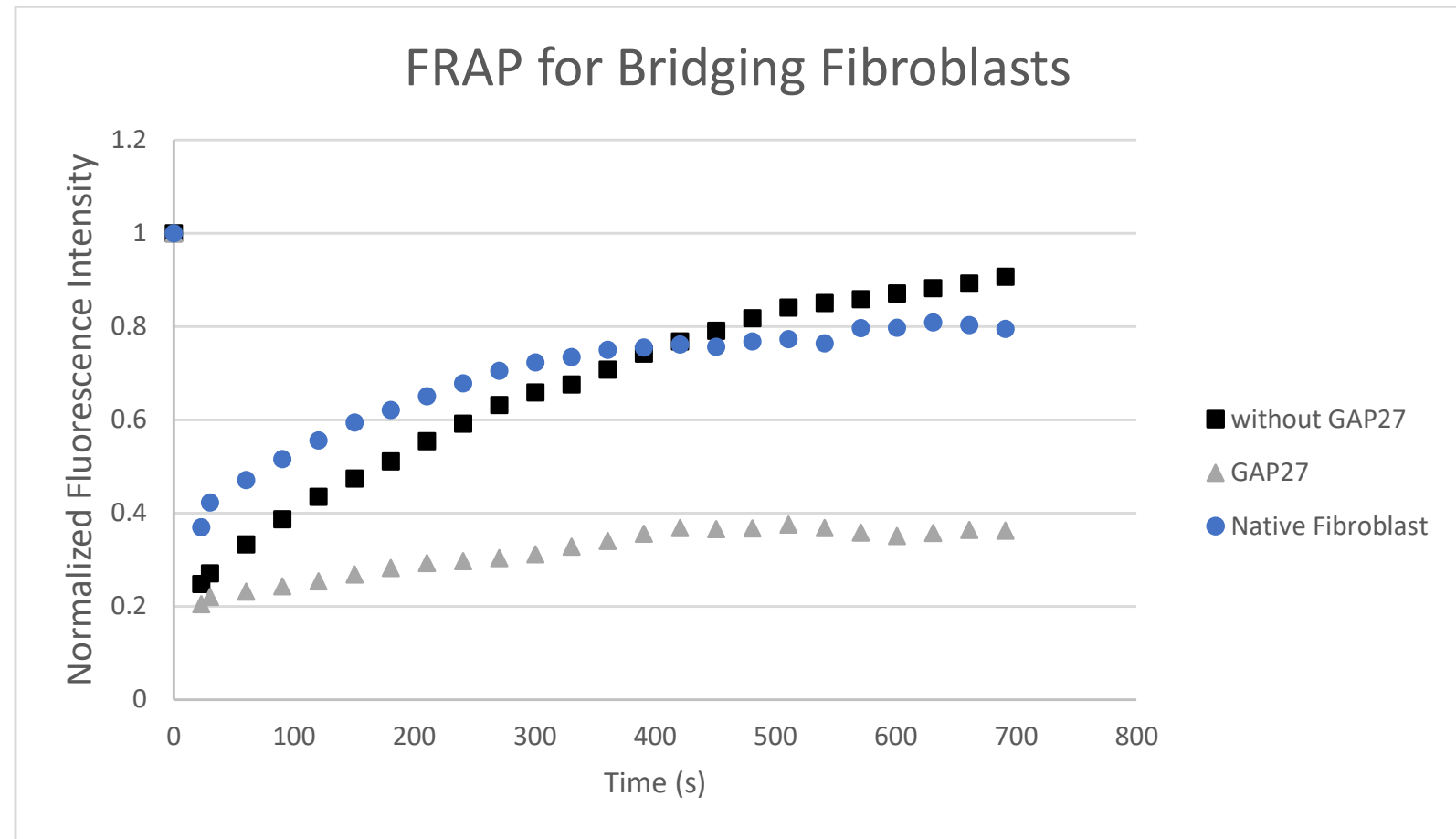

Figure 4.19: FRAP Fluorescence Intensities for Region 1 being the photobleached cell and Region 2 being the non-photobleached cell.

Many studies demonstrate a platform that can direct cellular attachment to a surface, however, no other studies found demonstrate the ability to direct cellular growth and maintain a communication network [6]-[8], [17], [20]-[39]. Additionally, attempts at cellular communication platforms focus mainly on the use of cell signaling to identify communication [102], [104]. The presented platform demonstrates the ability to do both of these tasks. 


\section{Recommendations and Conclusions}

\subsection{Recommendations}

The fabrication method demonstrated was completed with manual control on the movement and focusing of the microscope system. This resulted in a great deal of variability and why some shapes are not identical. Any additional testing should incorporate an automated system.

Following this, the next step should involve investigating the ability of nanoscale verse microscale wrinkle effects on cellular response. The specific results observed occurred during smooth and wrinkled morphologies. However, by altering the wavelength, it should be determined on this platform the point when the cellular response observed diminishes. Thus, identifying at what wavelength the cell stops detecting the wrinkled morphology.

Additionally, a cellular network designed to a specific post design should be tested. For example creating a design for a linear line which consists of post to post spacing at the correct distance for cellular bridging, but row spacing past the post of critical bridging distance, Figure 5.1. This would prevent bridging in one direction and allow bridging in another direction, or directed growth control. The potential addition of cell repellant to the surface would increase cellular interactions with the posts and prevent cellular attachment in a design where cells are no desired, such as the basement surface. 


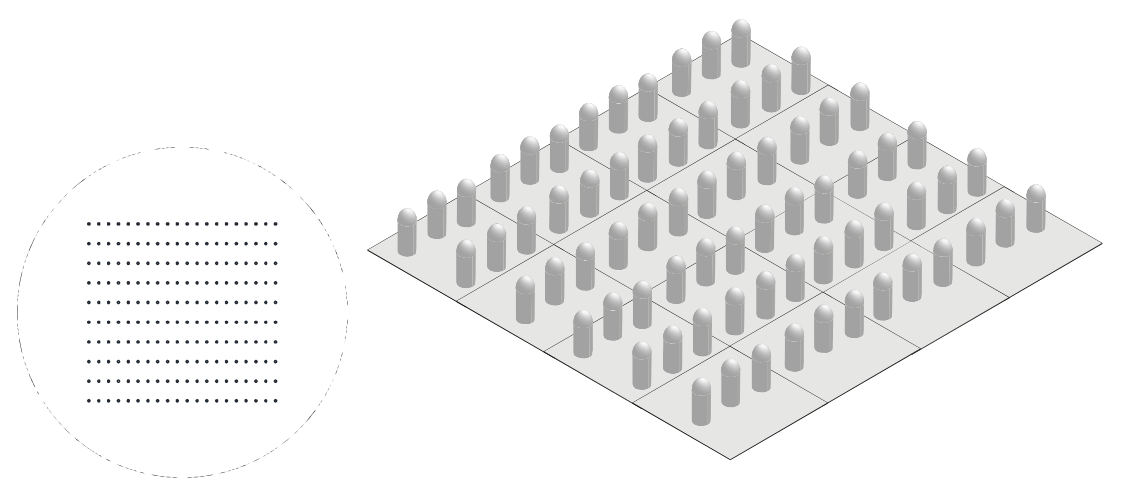

Figure 5.1: Potential new photomask design to create posts with a distance between

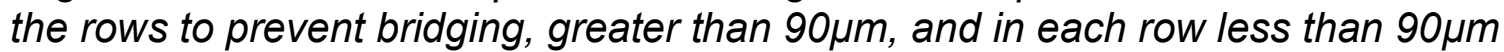
between each post. This would lead to lines of cells.

However, this platform is currently optimized only for bovine fibroblasts. Literature exemplifies that each individual cell has an ideal environment due to the wide variety of size and shape of cells. Thus, testing the platform with additional cell types is a key milestone for this platform. A large potential of applications exist when discussing the incorporation of other cell types. For example, ligament reconstruction, by creating a tissue engineered ligament by manufacturing fibers containing fibroblasts can be used to replace damaged ligaments such as the ligaments in the knee including the predominant ACL rupture. Alternatively, Nerve regeneration, applications to neurons to create a functional network which can be used as a biological model to understand more about this cell type would greatly benefit neuro based research.

It is hypothesised that a cellular mechanism is responsible for regulating these observed behaviors in response to topographical cues. The cellular mechanism underlying cell responses to physical cues are thought to depend on a mechanism similar to mechanotranduction. The next jump in research is to determine this 
underlying mechanisms and to induce these behaviors into the three dimensional environment.

On a more basic science level, cellular communication does not have a good model to control different aspects of cellular communication. The potential to create a cellular communication model to study the in-depth kinetics of cellular communications is a potential application.

On issue presented was the slight reflourscence of Calcein AM during the FRAP with GAP27 should be examined. Some literature supports that Calcein AM permeates both cxn43 and cxn37[103]. Thus the addition of an inhibitor for cxn37 may reduce the reflourscence. Additionally, other explanations as to the photobleaching effect should be explored such as, reflourscence from within the own cell from somewhere that was not photobleached.

\subsection{Conclusion}

In the present study, a cell culture platform was developed and optimized for culturing a cellular communication network between bovine fibroblasts. At the present, there have been negligible cellular communication networks created investigating and producing a viable network model. The results of the present study add to the available literature in tunable photolithography literature, nonplanar cell culture platforms, cellular response to topography, and cellular communication networks. These results may be used by other researchers to guide future work in these areas. The significant result from this research was the ability to create a directed growth cellular network that presented the ability to communicated which may help to specifically tailor new models to study cellular communication. 
Ultimately, this project will provide a platform that provides spatial organization and alignment while preserving the communication which occurs in-vivo. 


\section{Reference List}

[1] M.-H. Kim, M. Park, K. Kang, and I. S. Choi, "Neurons on nanometric topographies: insights into neuronal behaviors in vitro," Biomater. Sci., vol. 2, no. 2, p. 148, 2014.

[2] F. C. Su, C. C. Wu, and S. Chien, "Review: Roles of microenvironment and mechanical forces in cell and tissue remodeling," J. Med. Biol. Eng., vol. 31, no. 4, pp. 233-244, 2011.

[3] G. Bugnicourt, J. Brocard, A. Nicolas, and C. Villard, "Nanoscale surface topography reshapes neuronal growth in culture," Langmuir, vol. 30, no. 15, pp. 4441-4449, 2014.

[4] "Mechanobiology in harness," Nat. Mater., vol. 13, no. 6, pp. 531-531, May 2014.

[5] J. Y. Lim and H. J. Donahue, "Cell sensing and response to micro- and nanostructured surfaces produced by chemical and topographic patterning.," Tissue Eng., vol. 13, no. 8, pp. 1879-1891, Aug. 2007.

[6] M. J. Dalby, M. O. Riehle, S. J. Yarwood, C. D. W. Wilkinson, and A. S. G. Curtis, "Nucleus alignment and cell signaling in fibroblasts: Response to a micro-grooved topography," Exp. Cell Res., vol. 284, no. 2, pp. 274-282, Apr. 2003.

[7] N. M. Dowell-Mesfin, M. Abdul-Karim, a M. P. Turner, S. Schanz, H. G. Craighead, B. Roysam, J. N. Turner, and W. Shain, "Topographically modified surfaces affect orientation and growth of hippocampal neurons.," J. Neural Eng., vol. 1, no. 2, pp. 78-90, Jun. 2004.

[8] E. F. Petersen, R. G. S. Spencer, and E. W. McFarland, "Microengineering neocartilage scaffolds.," Biotechnol. Bioeng., vol. 78, no. 7, pp. 801-804, Jun. 2002.

[9] B. Palsson and S. Bhatia, Tissue Engineering. New Jersey: Pearson, 2009.

[10] V. Maruthamuthu, "Molecular mechanisms of cell adhesion," University of Illinois at Urbana, 2009.

[11] E. Stratakis, a Ranella, and C. Fotakis, "Biomimetic micro-nanostructured functional surfaces for microfluidic and tissue engineering applications.," Biomicrofluidics, vol. 5, no. 1, p. 13411, Jan. 2011.

[12] J. S. Fraser, J. D. Gross, and N. J. Krogan, "From Systems to Structure: Bridging Networks and Mechanism," Mol. Cell, vol. 49, no. 2, pp. 222-231, 2013.

[13] W. K. Lee, C. J. Engel, M. D. Huntington, J. Hu, and T. W. Odom, "Controlled Three-Dimensional Hierarchical Structuring by Memory-Based, Sequential Wrinkling," Nano Lett., vol. 15, no. 8, pp. 5624-5629, 2015.

[14] Y. Shao and J. Fu, "Integrated Micro/nanoengineered Functional Biomaterials for Cell Mechanics and Mechanobiology: A Materials Perspective," Adv. Mater. , vol. 26, no. 10, pp. 1494-1533, 2014.

[15] S. H. Hong, K. S. Han, K. J. Byeon, H. Lee, and K. W. Choi, "Fabrication of sub$100 \mathrm{~nm}$ sized patterns on curved acryl substrate using a flexible stamp," Jpn. J. Appl. Phys., vol. 47, no. 5, pp. 3699-3701, 2008.

[16] B. Farshchian, A. Amirsadeghi, S. M. Hurst, J. Wu, J. Lee, and S. Park, "Soft UVnanoimprint lithography on non-planar surfaces," Microelectron. Eng., vol. 88, no. 11, pp. 3287-3292, 2011.

[17] M. Ghibaudo, L. Trichet, J. Le Digabel, A. Richert, P. Hersen, and B. Ladoux, 
"Substrate topography induces a crossover from 2D to 3D behavior in fibroblast migration," Biophys. J., vol. 97, no. 1, pp. 357-368, Jul. 2009.

[18] W. a. Loesberg, J. te Riet, F. C. M. J. M. van Delft, P. Schön, C. G. Figdor, S. Speller, J. J. W. a van Loon, X. F. Walboomers, and J. a. Jansen, "The threshold at which substrate nanogroove dimensions may influence fibroblast alignment and adhesion," Biomaterials, vol. 28, no. 27, pp. 3944-3951, 2007.

[19] L. Wang, C. Lin, L. Yang, J. Zhang, and J. Zheng, "Preparation of nano/microscale column-like topography on PDMS surfaces via vapor deposition:

Dependence on volatility solvents," Appl. Surf. Sci., vol. 258, no. 1, pp. 265-269, Oct. 2011.

[20] E. Costa Martínez, J. L. Escobar Ivirico, I. Muñoz Criado, J. L. Gómez Ribelles, M. Monleón Pradas, and M. Salmerón Sánchez, "Effect of poly(L-lactide) surface topography on the morphology of in vitro cultured human articular chondrocytes," J. Mater. Sci. Mater. Med., vol. 18, no. 8, pp. 1627-1632, Aug. 2007.

[21] J. Shaik, J. Shaikh Mohammed, M. J. McShane, and D. K. Mills, "Chondrocyte Behavior on Micropatterns Fabricated Using Layer-by-Layer Lift-Off: Morphological Analysis," J. Med. Eng., vol. 2013, pp. 1-12, 2013.

[22] J. Szivek, D. Wiley, L. Cox, D. Harris, D. Margolis, and W. Grana, "Stem Cells Grown in Dynamic Culture on Micropatterned Surfaces can be used to Engineer Cartilage-like Tissue," in 53rd Annual Meeting of the Orthopaedic Research Society, 2007.

[23] R. Barbucci, P. Torricelli, M. Fini, D. Pasqui, P. Favia, E. Sardella, R. D’Agostino, and R. Giardino, "Proliferative and re-defferentiative effects of photo-immobilized micro-patterned hyaluronan surfaces on chondrocyte cells," Biomaterials, vol. 26, no. 36, pp. 7596-7605, Dec. 2005.

[24] F. Brétagnol, L. Ceriotti, M. Lejeune, A. Papadopoulou-Bouraoui, M. Hasiwa, D. Gilliland, G. Ceccone, P. Colpo, and F. Rossi, "Functional Micropatterned Surfaces by Combination of Plasma Polymerization and Lift-Off Processes," Plasma Process. Polym., vol. 3, no. 1, pp. 30-38, Jan. 2006.

[25] M. Nikkhah, F. Edalat, S. Manoucheri, and A. Khademhosseini, "Engineering microscale topographies to control the cell-substrate interface," Biomaterials, vol. 33, no. 21, pp. 5230-5246, Jul. 2012.

[26] M. D. Boehler, S. S. Leondopulos, B. C. Wheeler, and G. J. Brewer, "Hippocampal networks on reliable patterned substrates," J. Neurosci. Methods, vol. 203, no. 2, pp. 344-353, Jan. 2012.

[27] S. Morelli, S. Salerno, A. Piscioneri, B. J. Papenburg, A. Di Vito, G. Giusi, M. Canonaco, D. Stamatialis, E. Drioli, and L. De Bartolo, "Influence of micropatterned PLLA membranes on outgrowth and orientation of hippocampal neurites," Biomaterials, vol. 31, no. 27, pp. 7000-7011, Sep. 2010.

[28] a Béduer, L. Vaysse, E. Flahaut, F. Seichepine, I. Loubinoux, and C. Vieu, "Multiscale engineering for neuronal cell growth and differentiation," Microelectron. Eng., vol. 88, no. 8, pp. 1668-1671, Aug. 2011.

[29] a Beduer, C. Vieu, F. Arnauduc, J. C. Sol, I. Loubinoux, and L. Vaysse, "Engineering of adult human neural stem cells differentiation through surface micropatterning," Biomaterials, vol. 33, no. 2, pp. 504-514, Jan. 2012.

[30] E. M. Darling, P. E. Pritchett, B. a. Evans, R. Superfine, S. Zauscher, and F. 
Guilak, "Mechanical properties and gene expression of chondrocytes on micropatterned substrates following dedifferentiation in monolayer," Cell. Mol. Bioeng., vol. 2, no. 3, pp. 395-404, Aug. 2009.

[31] C. C. Berry, G. Campbell, A. Spadiccino, M. Robertson, and A. S. G. Curtis, "The influence of microscale topography on fibroblast attachment and motility," Biomaterials, vol. 25, no. 26, pp. 5781-5788, Nov. 2004.

[32] H. Jeon, H. Hidai, D. J. Hwang, K. E. Healy, and C. P. Grigoropoulos, "The effect of micronscale anisotropic cross patterns on fibroblast migration," Biomaterials, vol. 31, no. 15, pp. 4286-4295, May 2010.

[33] C. Luo, L. Liu, X. Ni, L. Wang, S. M. Nomura, Q. Ouyang, and Y. Chen, "Differentiating stem cells on patterned substrates for neural network formation," Microelectron. Eng., vol. 88, no. 8, pp. 1707-1710, Aug. 2011.

[34] C. C. Berry, M. J. Dalby, D. McCloy, and S. Affrossman, "The fibroblast response to tubes exhibiting internal nanotopography," Biomaterials, vol. 26, no. 24, pp. 4985-4992, Aug. 2005.

[35] M. Ghibaudo, J.-M. Di Meglio, P. Hersen, and B. Ladoux, "Mechanics of cell spreading within 3D-micropatterned environments.," Lab Chip, vol. 11, no. 5, pp. 805-812, Mar. 2011.

[36] P. Reemann, T. Kangur, M. Pook, M. Paalo, L. Nurmis, I. Kink, O. Porosaar, K. Kingo, E. Vasar, S. Kõks, V. Jaks, and M. Järvekülg, "Fibroblast growth on microand nanopatterned surfaces prepared by a novel sol-gel phase separation method.," J. Mater. Sci. Mater. Med., vol. 24, no. 3, pp. 783-92, Mar. 2013.

[37] D. Baker, X. Liu, H. Weng, C. Luo, and L. Tang, "Fibroblast/fibrocyte: surface interaction dictates tissue reactions to micropillar implants," Biomarcomolecules, vol. 12, no. 4, pp. 997-1005, 2011.

[38] A. Ruiz, L. Buzanska, D. Gilliland, H. Rauscher, L. Sirghi, T. Sobanski, M. Zychowicz, L. Ceriotti, F. Bretagnol, S. Coecke, P. Colpo, and F. Rossi, "Microstamped surfaces for the patterned growth of neural stem cells," Biomaterials, vol. 29, no. 36, pp. 4766-4774, Dec. 2008.

[39] J. L. Tan, J. Tien, D. M. Pirone, D. S. Gray, K. Bhadriraju, and C. S. Chen, "Cells lying on a bed of microneedles: an approach to isolate mechanical force.," Proc. Natl. Acad. Sci. U. S. A., vol. 100, no. 4, pp. 1484-1489, 2003.

[40] R. G. Harrison, "The reaction of embryonic cells to solid structures," J. Exp. Zool., vol. 17, no. 4, pp. 521-544, 1914.

[41] Y. Xia and G. M. Whitesides, "Soft Lithography," Annu. Rev. Mater. Sci., vol. 28, no. 1, pp. 153-184, 1998.

[42] Z. Li, J. A. Dranoff, E. P. Chan, M. Uemura, J. S??vigny, and R. G. Wells, "Transforming growth factor-?? and substrate stiffness regulate portal fibroblast activation in culture," Hepatology, vol. 46, no. 4, pp. 1246-1256, 2007.

[43] R. Mcmurray, M. J. Dalby, and N. Gadegaard, "Nanopatterned Surfaces for Biomedical Applications," no. Vieu 2000, 2004.

[44] A. D. Jenkins, P. Kratochvíl, R. F. T. Stepto, and U. W. Suter, "Glossary of basic terms in polymer science (IUPAC Recommendations 1996)," Pure Appl. Chem., vol. 68, no. 12, pp. 2287-2311, 1996.

[45] A. N. G. Gent, Alan N, R. P. Campion, M. D. Ellul, R. H. Finney, G. J. L. G. R. Hamed, D. L. Hertz, Jr., F. O. James, J. G. S. T. S. Miller, K. W. Scott, P. M. 
Sheridan, and O. H. Y. A. Stevenson, T. Sueyasu, A. G. Thomas, C. Wang, "Contents Engineering with Rubber," Eng. with Rubber How to Des. Rubber Components, 2001.

[46] S. J. Bryant, C. R. Nuttelman, and K. S. Anseth, "Cytocompatibility of UV and visible light photoinitiating systems on cultured NIH/3T3 fibroblasts in vitro," $J$. Biomater. Sci. Polym. Ed., vol. 11, no. 5, pp. 439-457, 2000.

[47] M. W. Tibbitt and K. S. Anseth, "Hydrogels as extracellular matrix mimics for 3D cell culture," Biotechnology and Bioengineering, vol. 103, no. 4. pp. 655-663, 2009.

[48] T. R. Hoare and D. S. Kohane, "Hydrogels in drug delivery: Progress and challenges," Polymer, vol. 49, no. 8. pp. 1993-2007, 2008.

[49] J. L. Drury and D. J. Mooney, "Hydrogels for tissue engineering: Scaffold design variables and applications," Biomaterials, vol. 24, no. 24. pp. 4337-4351, 2003.

[50] D. Castro, P. Ingram, R. Kodzius, D. Conchouso, E. Yoon, and I. G. Foulds, "Characterization of solid UV cross-linked PEGDA for biological applications," in Proceedings of the IEEE International Conference on Micro Electro Mechanical Systems (MEMS), 2013, pp. 457-460.

[51] N. Hakimi, "Single-Step Synthesis of Three-Dimensional Particles Using NonUniform Uv Polymerization," Ryerson University, 2014.

[52] K. Matyjaszewski and T. P. Davis, Handbook of Radical Polymerization, vol. 125, no. 11. 2002.

[53] D. Dendukuri, P. Panda, R. Haghgooie, J. M. Kim, T. A. Hatton, and P. S. Doyle, "Modeling of oxygen-inhibited free radical photopolymerization in a PDMS microfluidic device," Macromolecules, vol. 41, no. 22, pp. 8547-8556, 2008.

[54] M. Li, N. Hakimi, R. Perez, S. Waldman, J. a. Kozinski, and D. K. Hwang, "Microarchitecture for a Three-Dimensional Wrinkled Surface Platform," Adv. Mater., p. n/a-n/a, 2015.

[55] D. Dendukuri and P. S. Doyle, "The synthesis and assembly of polymeric microparticles using microfluidics," Adv. Mater., vol. 21, no. 41, pp. 4071-4086, Nov. 2009.

[56] N. Hakimi, S. S. H. Tsai, C. H. Cheng, and D. K. Hwang, "One-step twodimensional microfluidics-based synthesis of three-dimensional particles," Adv. Mater., vol. 26, no. 9, pp. 1393-1398, 2014.

[57] B. Li, Y.-P. Cao, X.-Q. Feng, and H. Gao, "Mechanics of morphological instabilities and surface wrinkling in soft materials: a review," Soft Matter, vol. 8, no. 21, p. 5728, 2012.

[58] M. Nania, O. K. Matar, and J. T. Cabral, "Frontal vitrification of PDMS using air plasma and consequences for surface wrinkling," Soft Matter, pp. 3067-3075, 2015.

[59] S. Lin, E. K. Lee, N. Nguyen, and M. Khine, "Thermally-induced miniaturization for micro- and nanofabrication: progress and updates.," Lab Chip, vol. 14, no. 18, pp. 3475-88, 2014.

[60] J. Y. Chung, A. J. Nolte, and C. M. Stafford, "Surface wrinkling: A versatile platform for measuring thin-film properties," Advanced Materials, vol. 23, no. 3. pp. 349-368, 2011.

[61] S. P. Bhawalkar, J. Qian, M. C. Heiber, and L. Jia, "Development of a colloidal 
lithography method for patterning nonplanar surfaces," Langmuir, vol. 26, no. 22, pp. 16662-16666, 2010.

[62] J. Yin, X. Han, Y. Cao, and C. Lu, "Surface Wrinkling on Polydimethylsiloxane Microspheres via Wet Surface Chemical Oxidation.," Sci. Rep., vol. 4, p. 5710, 2014.

[63] J. Le Digabel, M. Ghibaudo, L. Trichet, A. Richert, and B. Ladoux, "Microfabricated substrates as a tool to study cell mechanotransduction," Med. Biol. Eng. Comput., vol. 48, no. 10, pp. 965-976, Oct. 2010.

[64] D. Daramola, "Fabrication of micro/nano-structured wrinkles through surface modifcation of poly(dimethylsiloxane)," 2015.

[65] N. Bowden, S. Brittain, and A. Evans, "Spontaneous formation of ordered structures in thin films of metals supported on an elastomeric polymer," Nature, vol. 393, no. May, pp. 146-149, 1998.

[66] C. Cao, H. F. Chan, J. Zang, K. W. Leong, and X. Zhao, "Harnessing localized ridges for high-aspect-ratio hierarchical patterns with dynamic tunability and multifunctionality," Adv. Mater., vol. 26, no. 11, pp. 1763-1770, 2014.

[67] C. C. Fu, A. Grimes, M. Long, C. G. L. Ferri, B. D. Rich, S. Ghosh, S. Ghosh, L. P. Lee, A. Gopinathan, and M. Khine, "Tunable nanowrinkles on shape memory polymer sheets," Adv. Mater., vol. 21, no. 44, pp. 4472-4476, 2009.

[68] J. L. Yagüe, J. Yin, M. C. Boyce, and K. K. Gleason, "Design of ordered wrinkled patterns with dynamically tuned properties," Phys. Procedia, vol. 46, pp. 40-45, 2013.

[69] C. Yu and H. Jiang, "Forming wrinkled stiff films on polymeric substrates at room temperature for stretchable interconnects applications," Thin Solid Films, vol. 519, no. 2, pp. 818-822, 2010.

[70] M. Bullough and Y. Cui, "A library of large-scale surface patterns induced by flame on elastomers," Soft Matter, vol. 8, no. 12, p. 3304, 2012.

[71] M. D. Huntington, C. J. Engel, A. J. Hryn, and T. W. Odom, "Polymer nanowrinkles with continuously tunable wavelengths," ACS Appl. Mater. Interfaces, vol. 5, no. 13, pp. 6438-6442, 2013.

[72] H. T. Evensen, H. Jiang, K. W. Gotrik, F. Denes, and R. W. Carpick, "Transformations in wrinkle patterns: Cooperation between nanoscale crosslinked surface layers and the submicrometer bulk in wafer-spun, plasma-treated polydimethylsiloxane," Nano Lett., vol. 9, no. 8, pp. 2884-2890, 2009.

[73] D. B. H. Chua, H. T. Ng, and S. F. Y. Li, "Spontaneous formation of complex and ordered structures on oxygen-plasma-treated elastomeric polydimethylsiloxane," Appl. Phys. Lett., vol. 76, no. 6, p. 721, 2000.

[74] W. Zhang, Y. Li, C. Lin, Q. I. An, C. Tao, Y. Gao, and G. Li, "Electrochemical Polymerization of Imidazolum-lonic Liquids Bearing a Pyrrole Moiety," J. Polym. Sci. Part A Polym. Chem., vol. 46, no. 12, pp. 4151-4161, 2008.

[75] B. Farshchian, S. Park, J. Choi, A. Amirsadeghi, J. Lee, and S. Park, "3D nanomolding for lab-on-a-chip applications," Lab Chip, vol. 12, no. 2, p. 4764, 2012.

[76] Z. Wang, D. Tonderys, S. E. Leggett, E. K. Williams, M. T. Kiani, R. Spitz Steinberg, Y. Qiu, I. Y. Wong, and R. H. Hurt, "Wrinkled, wavelength-tunable graphene-based surface topographies for directing cell alignment and 
morphology," Carbon N. Y., vol. 97, pp. 14-24, 2016.

[77] E. M. Levina, M. a Kharitonova, Y. a Rovensky, and J. M. Vasiliev, "Cytoskeletal control of fibroblast length: experiments with linear strips of substrate.," J. Cell Sci., vol. 114, pp. 4335-4341, 2001.

[78] A. Grosberg, P. W. Alford, M. L. McCain, and K. K. Parker, "Ensembles of engineered cardiac tissues for physiological and pharmacological study: heart on a chip.," Lab Chip, vol. 11, no. 24, pp. 4165-73, 2011.

[79] H.-N. Kim, S.-H. Lee, and K.-Y. Suh, "Controlled mechanical fracture for fabricating microchannels with various size gradients.," Lab Chip, vol. 11, no. 4, pp. 717-722, 2011.

[80] A. Pimpin and W. Srituravanich, "Reviews on micro- and nanolithography techniques and their applications," Eng. J., vol. 16, no. 1, pp. 37-55, Jan. 2012.

[81] Q. Hou, D. W. Grijpma, and J. Feijen, "Porous polymeric structures for tissue engineering prepared by a coagulation, compression moulding and salt leaching technique," Biomaterials, vol. 24, no. 11, pp. 1937-1947, 2003.

[82] L.-S. Wan, Z.-M. Liu, and Z.-K. Xu, "Surface engineering of macroporous polypropylene membranes," Soft Matter, vol. 5, no. 9, p. 1775, 2009.

[83] E. Schuh, "Chondrocyte response to matrix elasticity," University of Lubeck, 2010.

[84] T. T. Meckmongkol, R. Harmon, P. McKeown-Longo, and L. Van De Water, "The fibronectin synergy site modulates TGF-beta-dependent fibroblast contraction.," Biochem. Biophys. Res. Commun., vol. 360, no. 4, pp. 709-14, Sep. 2007.

[85] S. S. Chi, J. B. Rattner, P. Sciore, R. Boorman, and I. K. Y. Lo, "Gap junctions of the medial collateral ligament: Structure, distribution, associations and function," J. Anat., vol. 207, no. 2, pp. 145-154, 2005.

[86] M. Kuzma-Kuzniarska, C. Yapp, T. W. Pearson-Jones, A. K. Jones, and P. a Hulley, "Functional assessment of gap junctions in monolayer and threedimensional cultures of human tendon cells using fluorescence recovery after photobleaching.," J. Biomed. Opt., vol. 19, no. 1, p. 15001, 2014.

[87] C. J. Wierenga, "Studying synapses," Int. Innov., pp. 22-23.

[88] E. Perez-Costas, "Microscopy techniques and the study of synapses," ... Top. Microsc. ..., pp. 164-170, 2007.

[89] K. R. Kay, C. Smith, A. K. Wright, A. Serrano-Pozo, A. M. Pooler, R. Koffie, M. E. Bastin, T. H. Bak, S. Abrahams, K. J. Kopeikina, D. McGuone, M. P. Frosch, T. H. Gillingwater, B. T. Hyman, and T. L. Spires-Jones, "Studying synapses in human brain with array tomography and electron microscopy.," Nat. Protoc., vol. 8, no. 7, pp. 1366-80, 2013.

[90] M. Maes, S. C. Yanguas, J. Willebrords, and M. Vinken, "Models and methods for in vitro testing of hepatic gap junctional communication.," Toxicol. In Vitro, vol. 30, pp. 569-577, 2015.

[91] M. Abbaci, M. Barberi-Heyob, W. Blondel, F. Guillemin, and J. Didelon, "Advantages and limitations of commonly used methods to assay the molecular permeability of gap junctional intercellular communication," Biotechniques, vol. 45, no. 1, pp. 33-62, 2008.

[92] A. a. Gill, Í. Ortega, S. Kelly, and F. Claeyssens, "Towards the fabrication of artificial 3D microdevices for neural cell networks," Biomed. Microdevices, vol. 17, no. 2, 2015. 
[93] J. Koskela, "Light-induced biomaterial microfabrication for advanced cell culturing-a comparative study," p. 103, 2010.

[94] K. Ko, P. Arora, W. Lee, and C. McCulloch, "Biochemical and functional characterization of intercellular adhesion and gap junctions in fibroblasts.," $\mathrm{Am}$. J. Physiol. Cell Physiol., vol. 279, pp. C147-C157, 2000.

[95] L. R. J. Scarratt, B. S. Hoatson, E. S. Wood, B. S. Hawkett, and C. Neto, "Durable Superhydrophobic Surfaces via Spontaneous Wrinkling of Teflon AF," ACS Appl. Mater. Interfaces, p. acsami.5b12165, 2016.

[96] H.-C. Jeong, H.-G. Park, Y. H. Jung, J. H. Lee, B.-Y. Oh, and D.-S. Seo, "Tailoring the Orientation and Periodicity of Wrinkles Using Ion-Beam Bombardment," 2016.

[97] J. J. Yoon, S. H. Song, D. S. Lee, and T. G. Park, "Immobilization of cell adhesive RGD peptide onto the surface of highly porous biodegradable polymer scaffolds fabricated by a gas foaming/salt leaching method," Biomaterials, vol. 25 , no. 25 , pp. 5613-5620, 2004.

[98] D. Falconnet, G. Csucs, H. Michelle Grandin, and M. Textor, "Surface engineering approaches to micropattern surfaces for cell-based assays," Biomaterials, vol. 27, no. 16, pp. 3044-3063, Jun. 2006.

[99] Q. Cheng, K. Komvopoulos, and S. Li, "Surface chemical patterning for long-term single-cell culture," J. Biomed. Mater. Res. - Part A, vol. 96 A, no. 3, pp. 507-512, 2011.

[100] D. Lehnert, B. Wehrle-Haller, C. David, U. Weiland, C. Ballestrem, B. A. Imhof, and M. Bastmeyer, "Cell behaviour on micropatterned substrata: limits of extracellular matrix geometry for spreading and adhesion.," J. Cell Sci., vol. 117, no. Pt 1, pp. 41-52, 2004.

[101] R. D. Sochol, A. T. Higa, R. R. R. Janairo, S. Li, and L. Lin, "Unidirectional mechanical cellular stimuli via micropost array gradients," Soft Matter, vol. 7, no. 10, p. 4606, 2011.

[102] M. G. Rubashkin, G. Ou, and V. M. Weaver, "Deconstructing signaling in three dimensions," Biochemistry, vol. 53, no. 13, pp. 2078-2090, 2014.

[103] J. Yang, R. L. Darley, M. Hallett, and W. H. Evans, "Low Connexin ChannelDependent Intercellular Communication in Human Adult Hematopoietic Progenitor/Stem Cells: Probing Mechanisms of Autologous Stem Cell Therapy," Cell Commun. Adhes., vol. 16, no. 5, pp. 138-145, 2009.

[104] X. Song, B. Kong, and D. Li, "A new tool for probing of cell-cell communication: Human embryonic germ cells inducing apoptosis of SKOV3 ovarian cancer cells on a microfluidic chip," Biotechnol. Lett., vol. 30, no. 9, pp. 1537-1543, 2008. 